Jovita Brüning, Marie Mückstein, Dietrich Manzey

\title{
Multitasking strategies make the difference: Separating processing-code resources boosts multitasking efficiency when individuals prefer to interleave tasks in free concurrent dual tasking
}

Journal article | Accepted manuscript (Postprint)

This version is available at https://doi.org/10.14279/depositonce-11183

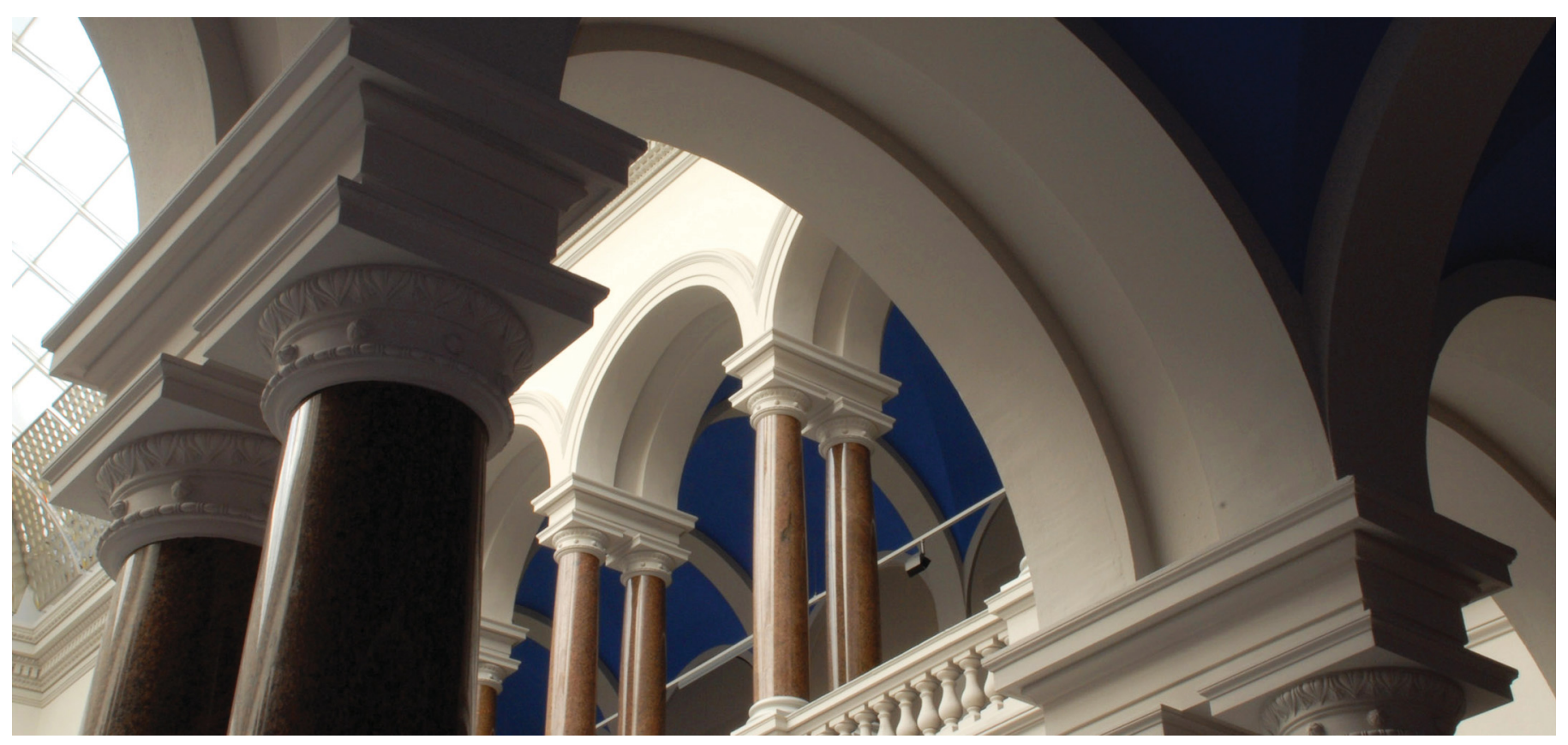

(C) American Psychological Association, 2020. This paper is not the copy of record and may not exactly replicate the authoritative document published in the APA journal. Please do not copy or cite without author's permission. The final article is available, upon publication, at:

https://doi.org/10.1037/xhp0000865

Brüning, J., Mückstein, M., \& Manzey, D. (2020). Multitasking strategies make the difference: Separating processing-code resources boosts multitasking efficiency when individuals prefer to interleave tasks in free concurrent dual tasking. Journal of Experimental Psychology: Human Perception and Performance, 46 (12), 1411-1433. https://doi.org/10.1037/xhp0000865 
Multitasking Strategies Make the Difference: Separating Processing Code Resources Boosts Multitasking Efficiency When Individuals Prefer to Interleave Tasks in Free Concurrent Dual-Tasking

\author{
Jovita Brüninga, b, Marie Mückstein ${ }^{\mathrm{a}} \&$ Dietrich Manzey ${ }^{\mathrm{a}}$
}

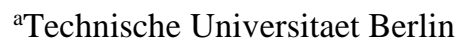

Work, Engineering and Organizational Psychology

Marchstrasse 12, F7

10587 Berlin

Germany

email: jovita.bruening@tu-berlin.de marie.mueckstein@ipu-berlin.de dietrich.manzey@tu-berlin.de

`Corresponding author. Please correspond to: jovita.bruening@tu-berlin.de.

Word count: 16081

Raw data and stimulus material are available via the Open Science Framework at https://osf.io/x6n58/

\title{
Acknowledgements
}

This research was supported by grant DFG 3759/4-1 provided from the Deutsche Forschungsgemeinschaft to the third author. We are grateful to Marcus Bleil for his technical support. Further, we are grateful to Iring Koch and an anonymous reviewer for helpful comments on the article. 


\begin{abstract}
Previous research has shown that individuals differ with respect to their preferred strategies in self-organized multitasking: They either prefer to work on one task for long sequences before switching to another (blocking), prefer to switch repeatedly after short sequences (switching), or prefer to respond almost simultaneously after processing the stimuli of two concurrently visible tasks (response grouping). In two experiments, we tested to what extent the choice of strategy and related differences in multitasking efficiency were affected by the between-resource competition (Wickens, 2002) of two tasks to be performed concurrently in a self-organized manner. All participants performed a set of dual tasks that differed with respect to the kind of stimuli (verbal vs. spatial) and/or responses (manual vs. vocal). The choice of strategy was hardly affected, as most individuals persisted in their response strategy independent of the degree of resource competition. However, the efficiency of individuals preferring a switching or response grouping strategy increased especially when the reduction in resource competition was response-related (manual vs. vocal), leading even to considerable dual-tasking benefits under these circumstances. In contrast, individuals who preferred to block their responses did not achieve any considerable benefits (or costs) with either of the different dual tasks.
\end{abstract}

Keywords: divided attention; dual-tasking; individual differences; multiple resources; performance efficiency

\footnotetext{
"CAmerican Psychological Association, 2020. This paper is not the copy of record and may not exactly replicate the authoritative document published in the APA journal. Please do not copy or cite without author's permission. The final article is available, upon publication, at: https://doi.org/10.1037/xhp0000865"
} 


\section{Public Significance Statement}

This study highlights individual differences in multitasking. It shows that people differ consistently in how they self-organize their responses when they perform two tasks concurrently. Some individuals prefer to perform the tasks serially even in the multitasking situation, whereas others prefer to conduct the tasks in a more interleaving manner with frequent switches back and forth between tasks or by responding almost simultaneously. These preferences are independent of the task characteristics and seem to reflect rather a difference in cognitive style than in capability. However, depending on the similarity of tasks, the different response strategies can differ in efficiency. Individuals preferring to work sequentially show neither significant benefits nor costs of multitasking. In contrast, individuals preferring to work on both tasks in an interleaving manner can gain considerable multitasking benefits compared to single-task performance when the tasks demand different processing codes (verbal vs. spatial) and response modalities (manual vs. vocal). 
Multitasking Strategies Make the Difference: Separating Processing Code Resources Boosts Multitasking Efficiency When Individuals Prefer to Interleave Tasks in Free

\section{Concurrent Dual-Tasking}

In recent decades, numerous studies have shown that our cognitive abilities are severely limited when we perform several tasks simultaneously or in close succession (Koch et al., 2018; Pashler, 2000; Salvucci, 2013). Especially research in cognitive psychology has been fruitful in determining specific mechanisms underlying multitasking performance (Fischer \& Plessow, 2015; Monsell, 1996, 2003; Pashler, 1994a, 2000). However, multitasking has not only important theoretical implications for the basic cognitive architecture, but is also of practical significance, e.g., in dealing with new media technology or in road traffic (Caird et al., 2008; Horrey \& Wickens, 2006; Mccart et al., 2006; Parry \& Le Roux, 2019; van der Schuur et al., 2015). For example, imagine a team of paramedics, who received a call to help injured people at an emergency. They arrive at an accident scene after a car crash, which happened because one of the drivers was talking on the phone while driving. In a critically limited period, the paramedics must assess the scene of the accident, gather information about the number and severity of injuries, communicate with those affected or witnesses to the accident, and prepare for admission to the emergency room. To this end, they must successfully coordinate their performance of all tasks in a well-organized and timely manner.

In order to understand how humans cope with multiple task demands, what limits arise and how an effective coordination of multiple tasks can be achieved, two different lines of research have been pursued. The first one involves research from a perspective of cognitive psychology, which addresses specific mechanisms of multitasking based on highly controlled experimental settings involving simple stimuli and responses and knowledge of the exact time-course of events (Koch et al., 2018). The second one involves human factors research, which usually takes a much more holistic view of multitasking performance. Human factors 
research mainly addresses the overall efficiency of concurrent task performance using experimental paradigms that demand participants to perform two or more tasks concurrently, as best as possible, without making precise prescriptions on how to achieve this goal (Wickens et al., 2013). Although both research lines have provided specific valuable insights into multitasking, they also have blind spots. What is missing from the most common approaches to multitasking in cognitive psychology is that human cognition happens continuously and in real time in a self-organized manner. A separation of the processes into discrete time steps and clear instructions as to when to respond to certain tasks cannot fully capture the observable behavior in everyday multitasking scenarios. In contrast, the traditional view of human factors and human performance research on multitasking just investigates selforganized multitasking, sometimes even in natural settings, but usually neglects how exactly an individual achieves a certain degree of multitasking efficiency and which cognitive mechanisms drive the observed performance.

\section{Process and Response Organization as Demands in Multitasking}

The research presented here is located between these two lines of research and can be seen as an approach to link them. The basic idea underlying our approach is that selforganized concurrent performance of two or more tasks always involves two interwoven challenges, which we refer to as task processing and response organization, and that individuals differ systematically in the way how they address these challenges in multitasking.

Task processing refers to the mechanisms involved in the cognitive processing of multiple tasks (i.e., independent of overt responses), often discussed as cognitive control processes. Originally conceptualized as a unitary function (Baddeley, 1986), cognitive control is now understood as an umbrella term that incorporates various functions like conflict adaptation, cognitive flexibility or working memory (Braem et al., 2019; Koch et al., 2018; Oberauer et al., 2018). Thus, task processing in multitasking describes how cognitive processes are organized when coping with concurrent task demands and what sort of 
constraints arise, for example, with respect to serial and parallel processing. This aspect has always represented one important focus of multitasking research in cognitive psychology based on paradigms like task-switching (Jersild, 1927) or the psychological refractory-period (PRP) paradigm (Telford, 1931; Welford, 1952). The research based on these paradigms has revealed mechanism such as re-configuration of task sets (Rogers \& Monsell, 1995) or taskset inertia effects (Allport et al., 1994; Koch \& Allport, 2006) when switching between tasks, or a central bottleneck which at least impedes the parallel selection of responses (Pashler, 1994a; Tombu \& Jolicœur, 2003). All these mechanisms represent examples of constraints of internal process organization that arise when humans must cope with multiple tasks concurrently. Besides, there is evidence from both, the task-switching and PRP paradigm that individuals differ in the way how they cope with these constraints in multitasking. Some individuals prefer to process different tasks in a more or less strictly serial manner, whereas others use options of parallel or at least overlapping processing if possible (Brüning et al., 2020; Brüning \& Manzey, 2018; Jersild, 1927; Maquestiaux et al., 2008; Maquestiaux et al., 2018; Reissland \& Manzey, 2016; Ruthruff et al., 2006; Schumacher et al., 2001). The paradigms used to isolate the different mechanisms of task processing were all optimized for this purpose, mainly by providing fixed task sequences, by strictly controlling the timing of events, by using bivalent stimuli (i.e., stimuli which principally fit to both tasks to be performed concurrently or in close succession), and/or by using a predetermined schedule for responding to the different tasks (e.g., according to specific alternating patterns in task switching or by prescribed sequences of responses in PRP tasks).

The latter aspect, however, largely neglects the second important challenge of selforganized everyday multitasking, that is the own organization of overt responses to different tasks. Response organization can be regarded as the behavioral level of task coordination in multitasking. It describes how a person organizes the performance of more than two tasks across time, which is reflected in a sequence of behavioral responses to the different tasks 
(i.e., when and for how long a person works on each of the concurrent tasks). This is a typical demand of everyday tasks, where we often have to decide when it is useful to switch between tasks, as this is usually not explicitly signaled by the environment (Janssen et al., 2011; Kushleyeva et al., 2005; Payne et al., 2007). In order to investigate how this is achieved and how this is related to the way internal processes are organized, paradigms are needed that offer participants degrees of freedom for their own response organization.

A paradigm previously used for this purpose as well as in the present research is the free concurrent dual-tasking (FCDT) paradigm (Brüning et al., 2020; Reissland \& Manzey, 2016). It capitalizes on the typical concurrent dual-task paradigms of the 1970s and 80s (Navon \& Gopher, 1979) but combines them with a novel fine-grained analyses of the participants' observable response patterns. In the FCDT paradigm, participants perform two tasks at their own pace by organizing when and for how long they perform each of the given tasks. The stimuli of both tasks are presented in two separate task threads and only the stimulus of the task currently being performed is updated while the stimulus of the other task remains visible. Participants are instructed to maximize their performance. However, no specific instructions are given how this could be achieved and the participants are completely free in organizing their responses to the concurrent tasks over time. The only limitation is to respond to about the same number of stimuli per task over time and/or to spend about the same time on both task streams. Thereby, this paradigm provides higher degrees of freedom for an individual's own task organization than other frequently applied paradigms in taskswitching or dual-tasking research (see Kiesel et al., 2010; Pashler, 1994a, for reviews). Using the FCDT paradigm, we forgo part of the experimental control over the timing of events to allow individuals to explore and develop their own strategies for dealing with more than one task under given circumstances. At the same time, we keep enough experimental control to determine, at a sufficiently detailed level, how individuals succeed in interleaving and switching between different tasks when working in a more self-organized manner. 
Using this paradigm, Reissland and Manzey (2016; Experiment 2) and Brüning et al. (2020) provided evidence that individuals differ considerably in how they spontaneously organize their responses when performing two tasks. A substantial proportion of participants (35\% - 46\% individuals) preferred to block their responses to each of the two tasks, that is, to work for long sequences on one task before switching to another. Others spontaneously chose to interleave the two tasks, either by repeatedly switching between them after short sequences of task repetitions (31\% - 35\%), or by grouping their responses to the two tasks $(23 \%-29 \%)$. These different response patterns correspond to early but not further pursued observations reported by Damos and Wickens (1980) and Damos et al. (1983) based on a similar paradigm. They already distinguished a simultaneous, alternating and massed strategy of response organization in participants working concurrently on a classification and short-term memory task which closely resemble the findings of Reissland and Manzey (2016) and Brüning et al. (2020). Besides, similar differences were occasionally observed in task-switching and PRP research, reflected in individual variations in the strength of repetition bias or the use of response grouping (Kessler et al., 2009; Mittelstädt et al., 2018; Mittelstädt et al., 2019; Pashler \& Johnston, 1989; Ulrich \& Miller, 2008).

Moreover, it seems that differences in response organization do not emerge entirely independently of the preferred mode of task processing, as suggested by the study of Brüning et al. (2020). For probing individual preferences with respect to the mode of task processing (serial vs. overlapping) they used the task-switching with preview (TSWP) paradigm. In this paradigm, participants are required to work on two discrete tasks $\mathrm{A}$ and $\mathrm{B}$ in a prescribed order (e.g., AAABBB), directly corresponding to an alternating runs paradigm (Rogers \& Monsell, 1995). However, while working on the three repetitions of one task, the stimulus of the other task, which has to be responded to after the task switch, can already be seen. Thus, the participants always have a preview on the specific task to be performed after the switch, which they either can ignore or use for overlapping processing to optimize the upcoming task 
switch. Based on a detailed analysis of switch and mixing costs in this paradigm, individuals can be classified as serial or overlapping processors based on whether they use the preview or not. While serial processors produced switch costs corresponding to those usually observed in task-switching without preview, overlapping processors showed strikingly fast switching times actually reflecting switch benefits in contrast to costs (see already Jersild, 1927 for similar observations). However, most interesting in the present context, the mode of task processing as identified based on the TSWP paradigm was closely linked to the preferred style of response organization observed when the same individuals performed the FCDT. That is, the majority of individuals (67\%) preferred either the combination of serial processing and blocked responding or of overlapping processing and interleaved responding. However, these links were not perfect. This confirmed the assumption that the preferences at both levels of task coordination are strongly linked but do not seem to just represent two sides of the same coin. Just as, for example, in PRP tasks, the same serial process organization at the response selection stage can be associated with different types of response organizations, i.e., serial responding versus response grouping (Pashler, 1994b).

\section{Efficiency of Individual Response Organization Strategies}

The finding of individual differences in how multitasking is performed in terms of task processing and response organization immediately raises the question to what extent these differences are reflected in differences in multitasking efficiency. What type of task processing and response organization is best to achieve maximum multitasking performance?

In the research conducted, thus far, significant differences in multitasking efficiency only emerged depending on individual preferences regarding the mode of task processing (serial vs. overlapping) used in the TSWP paradigm. In this case, overlapping processors outperformed serial processors. Due to their overlapping processing of the two tasks, they achieved overall time gains which resulted in a higher task throughput when performing two 
tasks according to an alternating runs scheme (AAABBB), compared to the respective singletask performance (Brüning \& Manzey, 2018; Reissland \& Manzey, 2016, Experiment 1).

In contrast, in the FCDT paradigm no significant differences in multitasking efficiency depending on the different preferences for response organization strategies were found (Brüning et al., 2020; Reissland \& Manzey, 2016, Experiment 2). Here, it was irrelevant whether individuals blocked their responses to the two tasks by trying to separate and shield them as best as possible, or whether they applied a task-interleaving strategy (i.e., switching or response grouping). Descriptively, the blocking strategy even tended to result in better efficiency than the other strategies (Reissland \& Manzey, 2016, Exp. 2). This absence of significant differences in the multitasking efficiency is particularly surprising given the link between the strategies of response organization and modes of processing described above (Brüning et al., 2020). Consistent with this link, the question arises, why overlapping processors could realize a higher multitasking efficiency than serial processors in the more controlled TSWP, but could not transfer this benefit to their self-organized performance in FCDT when using a switching or response grouping strategy. This is especially puzzling as interleaving response strategies are inherently characterized by a higher number of task switches than the blocking strategy. Thus, they could be expected to benefit from time-gains achieved at task switches due to overlapping processing of the tasks. However, the benefits achieved with overlapping processing appeared to have been absorbed by other costs arising specifically in self-organized multitasking. In this context, an increased degree of task interleaving seemed to render the coordination of responses more demanding than a simple blocking strategy (Brüning et al., 2020). The latter assumption is in line with findings from voluntary task switching (Arrington \& Logan, 2004, 2005) and PRP research (De Jong, 1995; Kübler et al., 2018). They show that already the act of choosing which task to perform requires additional time-consuming control processes. These additional cognitive control demands could then result in less efficient interleaving strategies. 
One important factor that might have contributed to the costs involved in coordinating responses in the FCDT, and which might have prevented a higher degree of overlapping processing, is the similarity of tasks used in our previous research. The two studies, addressing the strategies' efficiency in self-organized dual tasking, included tasks that contained visually presented verbal material (e.g., digits and/or letters) and manual responses by keystroke (Brüning et al., 2020; Reissland \& Manzey, 2016, Experiment 2). However, coordinating concurrent performance of highly similar tasks might aggravate to process tasks in an overlapping manner and to coordinate responses to the tasks, e.g., due to high risks of task interference. In turn, less similar tasks might entail less dual-task interference that needs to be resolved, thus, rendering it easier to maintain the respective task-sets in parallel and to switch between them or to apply overlapping task processing. As a consequence, possible benefits of overlapping processing combined with interleaving strategies of response organization should increase, leading to a higher multitasking efficiency of switching or response grouping strategies compared to a blocking strategy in self-organized dual-tasking.

Such an impact of task similarity on the efficiency of overlapping processing and interleaving strategies of response organization would be directly in line with the multiple resource theory (MRT) proposed by Wickens (1980, 1984, 2002, 2008). Wickens assumed that the human information processing system corresponds to a composition of multiple resource pools and that different tasks demand these resources to a different degree depending on their specific task characteristics. Specifically, he proposed four dimensions of resources associated with different stages of processing (perceptual-cognitive vs. response-related) ${ }^{1}$, different processing codes (verbal vs. spatial), different perceptual modalities (visual vs. auditory) and different visual channels (ambient vs. focal). The predictions that follow from

\footnotetext{
${ }^{1}$ Note that the understanding of "stages" in the MRT (Wickens, 2002) is a somewhat more global one as in other models (e.g., Pashler, 1994a; Tombu \& Jolicœur, 2003). It refers to only two different resource pools, assuming that perceptual and cognitive (e.g., working memory) processes on the one hand and response processes on the other compete for different resources.
} 
MRT for concurrent task performance are straightforward: The more two tasks compete for similar processing resources, the higher are the risks of task interference and the need for cognitive control to reduce these risks (Wickens, 2002). As a result, a separation of resources demanded by tasks might also be expected to boost the ease and efficiency of overlapping processing and the related use of interleaving response strategies, whereas a high competition for resources might render serial processing and blocking of tasks at least as efficient.

\section{Current Research}

Based on this reasoning, the current research addresses the question to what extent the choice and efficiency of different strategies of response organization in self-organized multitasking would be determined by the degree of resource competition between tasks. In this respect, the dimension of processing codes proposed in the MRT (Wickens, 2002) appears particularly interesting because it allows to systematically vary resource competition at different processing stages. On the one hand, it distinguishes verbal versus spatial resources needed for perceptual-cognitive processing. This separation parallels the model of working memory developed by Baddeley $(1986,2002,2012)$ and is often associated with the functional specialization of the two cerebral hemispheres (Fried et al., 2014; Polson \& Friedman, 1988; Smith et al., 1996). In addition, it also differentiates between responserelated resources demanded by vocal (verbal) versus manual (spatial) responses. This latter extension is supported by early findings showing that tasks, which involve different response modalities, are performed with a higher efficiency than tasks sharing the same response modality (McLeod, 1977; Wickens et al., 1983; Wickens \& Liu, 1988). Overall, the dimension of processing codes, thus, refers to a distinction of resources on all three processing stages, i.e., perception, central processing and action (for an elaborate discussion of the importance of the processing code dichotomy see Wickens \& Liu, 1988). Furthermore, it has already been shown that the separation of the resources needed to perform two tasks in a dual-task combination along this dimension offers great potential to reduce dual-task costs 
and, thus, improve dual-task performance (Göthe et al., 2016; Polson \& Friedman, 1988; Schaeffner et al., 2018; Wickens et al., 1983).

Concerning the efficiency of different response strategies in self-organized multitasking, we assumed that task-interleaving strategies would be the more effective in dual-task performance, the less the two tasks compete for resources sharing the same processing code (verbal vs. spatial). Remember that interleaving strategies of response organization involve a higher number of task switches, which are essentially the basis for time gains due to overlapping processing (i.e., speeded responses at task switches). At the same time, however, these strategies are more complex than a simple blocking strategy and, thus, involve a higher demand for cognitive control and a higher risk of task-interference costs (Brüning et al., 2020). To the extent that a separation of resource demands facilitates overlapping processing, reduces risks of task interference and lowers demands on cognitive control, the net benefit (e.g., in terms of task throughput achieved) of these strategies should be increased. Conversely, individuals preferring the blocking response strategy only switch between the tasks a few times, so that naturally advantages of overlapping processing can only rarely be realized and switch costs can hardly accumulate. Therefore, the blocking strategy is not expected to produce significant differences in performance depending on the degree of resource competition.

In contrast to the expectations regarding the efficiency of the strategies, predictions to what extent the individual choice of a specific dual-tasking strategy would be affected by the degree of resource competition of two tasks were somewhat more difficult to derive, due to the lack of available empirical findings to date. However, there is some evidence that individuals can flexibly adjust the use of serial versus overlapping processing in PRP tasks based on instructions (Fischer \& Plessow, 2015; Lehle \& Hübner, 2009), and that they adapt their preference for the different modes of task processing in TSWP to the risk of betweentask crosstalk (Brüning \& Manzey, 2018). Combined with the finding that preferences for a 
certain mode of processing and preferences for different strategies of response organization are systematically linked (Brüning et al., 2020), at least some cautious assumptions can be made. In this vein, it could be expected that more individuals choose an interleaving strategy when the competition between the processing code resources in a dual task is low, as interleaving is likely to be more advantageous under these circumstances. By contrast, if the competition is high, it could be expected that more individuals choose a blocking strategy, given that the more complex interleaving strategies obviously do not provide a significant advantage in this case. However, such adaptational processes could also be reflected in an intensified use of the respective strategy depending on the between-task resource competition. For example, it could also be expected that switching is increased when different instead of similar processing code resources need to be allocated.

The hypotheses described were addressed in two consecutive experiments using the FCDT paradigm. In the first experiment, we tested whether the individual choices and the efficiency of different strategies of response organization in self-organized dual-tasking depend on whether the tasks compete for perceptual-cognitive resources in the processing code dimension (verbal vs. spatial). In the second experiment, we went even further by contrasting dual tasks consisting of component tasks that do or do not compete for perceptualcognitive resources, response-related resources or both types in the processing-code dimension. The latter included component tasks with resource profiles, which have been shown to be particularly compatible for multitasking in PRP and task-switching research (Göthe et al., 2016; Schaeffner et al., 2018). In addition, also two measures assessing individual differences in working memory and multitasking preferences were included in the second experiment, in order to explore possible links between these individual task characteristics and the choice of a given style of response organization. 


\section{Experiment 1}

With the first experiment, we aimed to determine whether the degree to which concurrently performed tasks compete for the same cognitive resources would affect the choice of specific response strategies and their efficiency. To this end, we developed four single-tasks that varied qualitatively in terms of whether they required verbal or spatial processing code resources according to the MRT (Wickens, 1984, 2002). Based on these tasks, four dual tasks were composed, which had to be performed in a self-organized manner according to the FCDT paradigm. Two of these dual tasks involved task combinations with high between-task resource competition, where both tasks to be performed concurrently competed for the same resources with respect to input modality (visual), the processing code needed for perceptual-cognitive processing of the tasks (either both verbal or both spatial), and output modality (manual responses). The other two dual tasks comprised two dual-task combinations where both tasks competed for the same resources regarding input modality (visual) and output modality (manual responses), but required different resources for perceptual-cognitive processing (verbal and spatial). Thus, they constituted dual-tasks with low between-task resource competition. We decided to use two combinations of dual tasks per condition, which essentially represented the same comparison, to ensure that the critical factor in testing the influence of resource competition is indeed the modulated variability of the processing code (i.e., spatial vs. verbal stimuli pairings), and not merely the particular features or rules of the tasks used.

\section{Method}

\section{Participants}

In the experiment, 48 volunteers took part. The dataset of two participants were excluded from the analysis due to high error rates $(\mathrm{ER} ;>20 \%)$ in at least one of the tasks. Thus, the final sample included 46 participants $(32$ female, mean age $=25.3$ years, standard 
deviation, $S D=3.2$ years, range $=18-32$ years). According to a power analysis (Faul et al., 2009), this sample size allows for detecting large effects with a power of .80 and an alpha of .05 , given our statistical approach to test the main working hypotheses for flexibility and efficiency of response organization strategies. All volunteers were either right-handed or ambidextrous, were able to speak German at native language level and had normal or corrected-to-normal vision. Furthermore, all experiments adhered to the standards set by the local ethics committee. They received 25 Euro or a course credit plus a monetary bonus for each correctly answered stimulus, which could add up to 10 Euro.

\section{Tasks}

Two verbal and two spatial tasks were used in the experiment. With respect to task rules, each set of two tasks consisted of one classification and one memory-search task. The verbal classification task required the participants to decide whether a presented pair of digits contained two digits of the same or different parity. The probability of same and different parity pairs was the same (50\%). In the verbal memory-search task two pairs of letters were presented for 10 seconds before each task block. The participants were asked to memorize those pairs. In the subsequent task block they decided whether the currently presented letter pair equals one of the memorized letter pairs. Target letter pairs were presented in half of the trials. Note that the participants were instructed to memorize the pairs of letters independently of the exact order of the letters. In the spatial classification task, pairs of three-dimensional (3D) objects were presented with the object to the right either representing the one on the left but rotated by $50^{\circ}$, or the object to the left but mirrored and rotated by $50^{\circ}$. Participants were asked to classify the pairs with respect to whether the right object was just rotated or rotated and mirrored, compared to the left object. The a priori probability of each kind of pair was the same (50\%). Finally, the spatial memory-search task required participants to memorize three simple two-dimensional patterns, which were presented in form of duplicates for 15 seconds prior to each task block. During the subsequent task block they indicated whether the 
currently presented pair of patterns was the same as one of the memorized pairs of patterns.

The target stimuli were presented in half of the trials. Based on these four tasks, four different dual tasks were defined (see Figure 1).

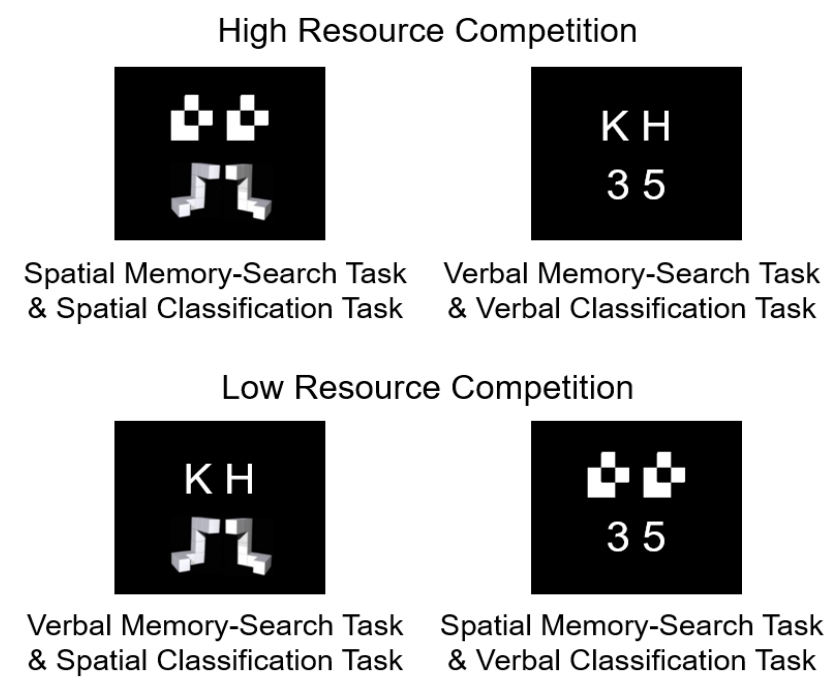

Fig. 1 Example of the four defined dual tasks. Each dual task comprised a classification- and a memory-search task. In the high resource competition condition both tasks demanded the same central processing code, either spatial (upper left side) or verbal (upper right side). In the low resource competition, both tasks demanded different central processing codes (a mixture of verbal and spatial tasks; lower row)

Each dual task consisted of a classification- and a memory-search task. Two of them involved tasks demanding the same central processing code (i.e., either both tasks are verbal, or both are spatial), whereas the other two dual tasks involved tasks demanding different central processing codes (i.e., a mixture of verbal and spatial tasks).

\section{Stimuli and Apparatus}

The experimental stimuli were displayed in light grey $(\mathrm{RGB}=245,245,245$; font size $=24 \mathrm{px})$ on black background $(\mathrm{RGB}=0,0,0)$ on an Acer LCD screen $(1280 \mathrm{x} 1024 \mathrm{px}$, sampling with $60 \mathrm{~Hz}$ ). They were presented in vertical arrangement with close spatial proximity $($ distance $=16 \mathrm{px})$, making concurrent perception of the two stimuli possible without eye movements. For the verbal classification task, the stimuli were drawn from a set 
of four pairs of digits with same parity $(24 ; 35 ; 68 ; 79)$ and four sets of digits with different parity $(29 ; 38 ; 47 ; 56)$. Stimuli for the verbal memory-search task were drawn from the consonants of the alphabet (B, C, D, F, G, H, J, K, L, M, N, P, Q, R, S, T, V, W, X, Y).

For the spatial classification task, task stimuli were composed based on four baseline objects, each paired with target objects either representing the same object but rotated by $50^{\circ}$ or representing a mirror image of the object rotated by $50^{\circ}$. This resulted in a set of eight possible task stimuli. The baseline objects were selected from the set of three-dimensional shapes introduced by Ganis and Kievit (2015). For the spatial memory-search task, a total of 18 patterns of randomly distributed squares along a 3 x 3 grid were used, selected from Patera (2018).

Stimulus presentation and response recording was controlled by a custom-made JAVA software running on an Intel Pentium (2.9 GHz, 8 GB RAM; Windows 7 Pro). In all blocks the stimuli of the currently performed task were shown until a response was recorded, immediately followed by the next stimulus (response-stimulus interval $=0 \mathrm{~ms}$ ). Participants responded by pressing predefined letters on a standard keyboard. The keys ' $\mathrm{K}$ ' and ' $\mathrm{L}$ ' were used with the index and middle finger of the right hand to respond to one task, and the keys ' $S$ ' and ' $A$ ' with fingers of the left hand for the other task, respectively. The task-hand assignment was counterbalanced between participants. The keys were marked with a blue, yellow, red or green color sticker to facilitate recognition.

\section{Procedure}

All volunteers participated in the experiment on three consecutive days. On all days, one to five participants worked simultaneously at independent PC workstations, separated by opaque screens. The first day included familiarization and practice of all single tasks, and the four dual tasks. During familiarization, instructions for each task were presented on the computer screen and could be read self-paced. On the second and third day, all participants were tested in two of the four dual-task combinations, respectively. The conditions of low 
versus high resource competition were tested on separate days. The order of conditions and according dual tasks performed per day were counterbalanced.

The different dual tasks were performed according to the scheme of the FCDT paradigm (Brüning et al., 2020; Reissland \& Manzey, 2016). The participants worked on both tasks in a self-organized manner, that is, they were free to decide when and for how long they wanted to work on each task, with the only constraint that they were instructed to not neglect or prefer one of the tasks compared to the other. For this purpose, the stimuli of the two tasks always were presented in proximity as two independent task streams. While a participant worked on one of the tasks, the stimuli of this task were replaced in a self-paced manner without any response-stimulus interval (RSI), while the stimulus of the other task remained. Only when the participant decided to switch to the other task and to begin performing it, the stimuli of this task were replaced while the last updated stimuli of the previously performed task remained. A scheme of an exemplary stimulus presentation of one of the dual tasks used in low-resource-competition condition of the experiment is shown in Figure 2.

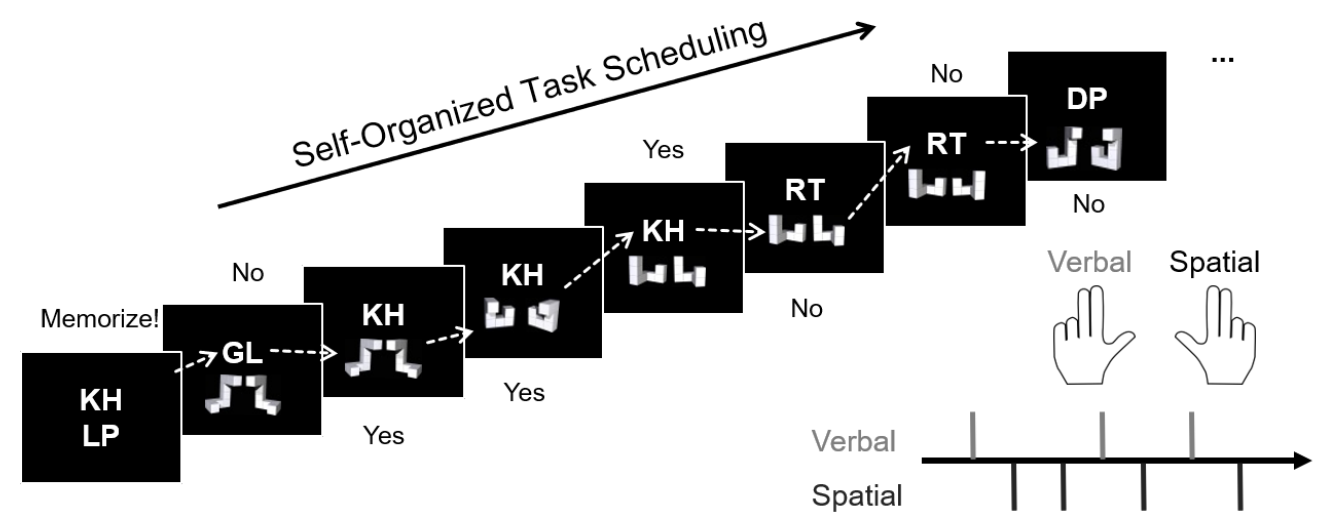

Fig. 2 Exemplary stimulus presentation of the low resource competition condition in free concurrent dual-tasking. Participants scheduled their responses in a self-organized manner indicated by a white arrow trajectory. The specific response pattern of both tasks, which are voluntarily produced, is depicted in the lower right corner

As can be seen from this figure, the dual-task blocks began with the simultaneous presentation of stimuli for both tasks. The participants then could decide freely which task to 
start with and how they preferred to organize their responses to the two tasks across time. Since responses to the two tasks came from different hands, the specific sequence of responses to the two tasks could then be derived post-hoc from the timeline of the response records. Accordingly, the number of task switches and task repetitions could vary between participants within and across conditions. The arrows in the figure describe a typical schedule of responses of a participant using what we refer to as a switching strategy. Note that the stimulus presentation of the FCDT paradigm also provides certain degrees of freedom at the level of task processing. As described above, while working on one of the tasks, the stimulus of the pending task was also visible. This arrangement principally allowed participants to apply either a serial or an overlapping mode of task processing (cf. Brüning \& Manzey, 2018), depending on whether they already started to process the stimulus of the respective pending task before switching to this task. Participants were not provided with any specific instructions regarding potential approaches of response organization or task processing.

Throughout the whole experiment, single-task and dual-task performances were assessed in runs with fixed time periods, and participants were always instructed to maximize their amount of correct responses in the given time, that is, to perform the tasks according to an optimum speed-accuracy balance. On the training day, each single-task was explained, including a 30 -s block of familiarization with the task, followed by a 60 -s practice block. In the course of the explanation of the spatial tasks, participants saw an overview of all upcoming stimuli of the respective task for $60 \mathrm{~s}$ to become familiarized with the stimulus material. Then, the participants practiced each single task for further $120 \mathrm{~s}$ to account for initial practice effects. Afterwards, the FCDT procedure was described in general and practiced with each dual-task combination in two 120-s practice blocks. Note that the order of tasks in the single-task training and the order of the dual tasks in the dual-task training were balanced across participants. 
On the test days, a short warm-up practice preceded the test phase including 30-s blocks per single task and a 60-s block of the according dual task. In the test phase, the data collection for each dual task included three runs. Every run comprised two 120-s dual-task blocks followed by one 60 -s block of each respective single task. The two single-task blocks were included to control for stability of single-task performance. Their order was counterbalanced across these blocks. The task stimuli of each block were randomly drawn from the stimulus sets of the respective tasks with the constraint that no stimulus would be directly repeated. At the end of every block, the participants were provided with feedback on the number of performed trials and the number and the percentage of correct responses of each task for five seconds. Short breaks of one and two minutes, were included between different task blocks and the two different dual-task conditions (days \#2 and \#3), respectively. Overall, each of the three sessions lasted about 1 hour.

\section{Design}

Based on their performance in the FCDT paradigm, all participants were categorized post-hoc regarding their strategy of response organization, i.e., as blocker, switcher, or response grouper. The groups of participants preferring the same strategy then served as three levels of the between-subject factor preferred strategy. Together with the within-subject factor resource competition, this resulted in a 2 (resource competition: low vs. high) x 3 (preferred strategy: blocker vs. switcher vs. response grouper) experimental design.

\section{Data Analyses}

Data structure and preprocessing. Three different trial types were considered when analyzing the data: single-task trials, repetition trials and switch trials. Repetition trials were defined as trials in which participants performed the same task as on the previous trial, switch trials included all trials in which participants performed a different task compared to the previous trial. Since the participants had the freedom to organize their responses to the two tasks in the dual-task conditions according to their preferred strategy, the type of a trial in the 
dual-task blocks was classified post hoc. For each trial type, the data of each participant were collapsed across tasks and experimental runs in the four different single tasks and dual tasks. This yielded 222 single-task trials $(S D=41)$ and 442 dual-task trials $(S D=96)$ per dual-task condition and participant on average. Note that the participants differed in the number of trials they performed because a fixed amount of time was necessary to calculate the "task throughput" (see section on multitasking efficiency). For the single-task trials and the different types of trials in dual-task blocks, the mean inter-response intervals (IRIs) of correct responses and mean error rates (ERs) were calculated for each participant. IRIs had to be used to assess response times because in dual-task blocks, due to the logic of the FCDT paradigm, one or more responses to the other task could be given within the interval between the stimulus and response of a given task. ERs were calculated as the number of incorrect responses compared to the total number of responses given per block. Regarding the identification of outliers at single-trial level, first, all trials with an IRI longer than five seconds were excluded. Subsequently, trials with an IRI slower than two SD from the participant's mean IRI in the according trial type were discarded. This resulted in an average of $4.54 \%$ of discarded single task trials $(S D=1.36)$ and $6.76 \%$ of discarded dual-task trials $(S D=3.03)$ per participant.

Identification of individually preferred strategies of response organization. Based on our previous research (Brüning et al., 2020; Reissland \& Manzey, 2016), we expected to distinguish three different strategies of response organization. In line with our categorization approach described in Brüning et al. (2020), these strategies were determined based on the individual switch rates and the distributions of IRIs in switch trials.

The switch rate was defined as the number of task switches related to the overall number of processed trials. Participants with a switch rate below $10 \%$ were classified as blocker as they obviously preferred to separate the performance of both tasks as much as possible even in dual-task trials. All participants with a switch rate higher than $10 \%$ and a 
unimodal distribution of IRIs in switch trials were classified as switcher. Here, the switch IRIs fluctuated rather uniformly around the mean IRI of switch trials. Response grouper were distinguished from the other groups based on a relatively high switch rate (> 50\%) along with a bimodal distribution of switch IRIs. Response groupers apply a strategy of withholding their responses until they have processed both tasks internally and then finally executed the according responses in close succession. Accordingly, they produce a characteristic pattern of relatively long and very short switch IRIs (for a similar approach of identifying response grouping see Strobach et al., 2014). In order to test whether the distribution of switch IRIs for participants showing high switch rates deviated from a unimodal distribution (i.e., being most likely bimodal) the Hartigan's dip test (Hartigan \& Hartigan, 1985) was used. Since this test is highly sensitive for signs that contradict unimodal distributions, we considered a $\mathrm{p}$-value of $\mathrm{p}$ $<.001$ as critical to reject unimodality. However, for all participants classified as response grouper this classification was always additionally confirmed by visual inspection of the distribution of switch IRIs.

In order to prove the reliability of this categorization procedure, we compared whether categorizations based on subsets of our data per condition yielded the same results. For this purpose, we conducted the categorization of each individual participant two times, each based on half of the data of the different dual-task conditions. All blocks of a given dual-task condition were split in successive 10 trial "chunks", which were numbered. Then, all even numbered chunks were used as one subset and all odd numbered chunks as a second subset for post-hoc classification of the individual response strategy. This allowed to evaluate the stability of categorization based on two halves of the data analog to the usual approach of split-half reliability assessments.

Analyses of multitasking efficiency. The multitasking efficiency of the different response strategies was assessed by the overall dual-tasking performance efficiency (ODTPE) measure proposed by Reissland and Manzey (2016) and refined by Brüning and Manzey 
(2018). The ODTPE relates the number of correct responses achieved per given time in dualtask blocks compared to baseline performance in single-task blocks, thus, representing a straightforward throughput measure. The higher the ODTPE scores, the better the performance in dual-task blocks relative to single-task blocks in terms of speed and accuracy of responding (net benefits: ODTPE > 0). In contrast, negative ODTPE scores reflect poorer performance in dual-task blocks compared to single-task blocks (net costs: ODTPE < 0). ODTPE scores $=0$ indicate a situation where concurrent performance of two tasks neither lead to performance costs nor benefits compared to typical single-task performance. A more detailed description of the ODTPE measure can be found in the appendix.

\section{Results}

\section{General Effect of Lowered Resource Competition}

At the level of the whole sample, the error rates were generally low with all singletask and dual-task error rates $<5 \%$ (see Supplemental Material 1 for more details on IRIs and ERs). Besides, the participants complied well with the instruction of equal task priority (Mean $=1.04, S D=0.17)$. In both between-task resource competition conditions, the mean switch rates and ODTPE scores differed only marginally (Table 1).

Table 1. Mean percentage and standard deviation (SD) of switch rate and ODTPE scores per condition of resource competition.

\begin{tabular}{lll}
\hline Condition of resource competition & Switch Rate (SD) & ODTPE (SD) \\
\hline high & $27.97(35.26)$ & $-0.16(7.91)$ \\
low & $29.91(35.20)$ & $1.06(10.78)$ \\
\hline
\end{tabular}

Contrasting these differences by t-tests, no significant effects of separation of processing-code resources were found, neither for multitasking efficiency, $t(45)=1.00, p=.323, d=0.19$, nor on the switch rate, $t(45)=1.76, p=.085, d=0.32$. However, the high standard deviations in the switch rates already point to considerable differences between individuals which were expected and are considered in detail in the following. 


\section{Reliability and Flexibility of Individual Multitasking Strategies}

Figure 3 illustrates the results of the split-half reliability assessments of our categorization procedure. As becomes evident, all participants could unambiguously and reliably be classified as either blocker, switcher or response grouper in all four dual tasks. Aggregated for both conditions of resource competition, the mean error rates of these subgroups again were $<5 \%$ and, therefore, neglected in the following analyses (see also Supplemental Material 1 for more details on IRIs and ERs).

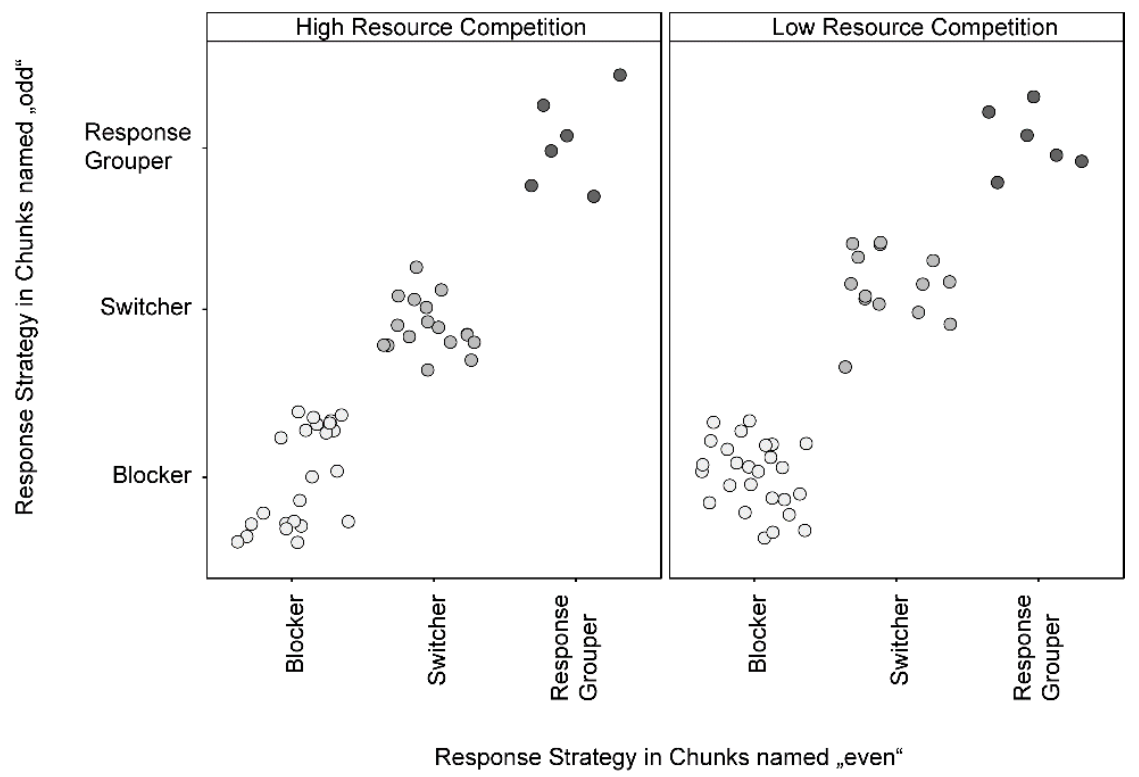

Fig. 3 Results of the split-half reliability assessments. Circles represent the classified response strategy for each individual based on aggregated data in "odd" versus "even" labeled chunks in the conditions of high/low resource competition (same/different processing-code). Data points are jittered for reasons of illustration

The two dual-task combinations that represented the same high versus low resource competition condition (i.e., verbal/verbal and spatial/spatial vs. twice verbal/spatial), each consisted of similar types of tasks (classification and memory-search). Thus, participants were expected to use essentially the same response organization strategies for dual tasks representing the same conditions of either low or high resource competition. Indeed, this held true for $n=43$ out of the 46 participants. However, in contrast to our expectation, a similar stability in the preference for a certain strategy of response organization was also found across 
the two conditions of resource competition. In fact, 41 individuals showed a stable strategy preference across the two conditions, whereas only two individuals changed their strategy dependent on the degree of resource competition across conditions. The according McNemar test, used to test for such changes, was not significant, $\chi^{2}(1, \mathrm{n}=43)=0.0, p=1$.

\section{Efficiency of Individual Multitasking Strategies}

Only the 41 individuals showing a stable preference within and between the conditions of high and low resource competition were included in the comparison of the multitasking efficiency achieved with the different strategies. Figure 4 shows the mean ODTPE scores of individuals preferring consistently the blocking, switching or response grouping strategy, averaged across the two dual tasks of each condition.

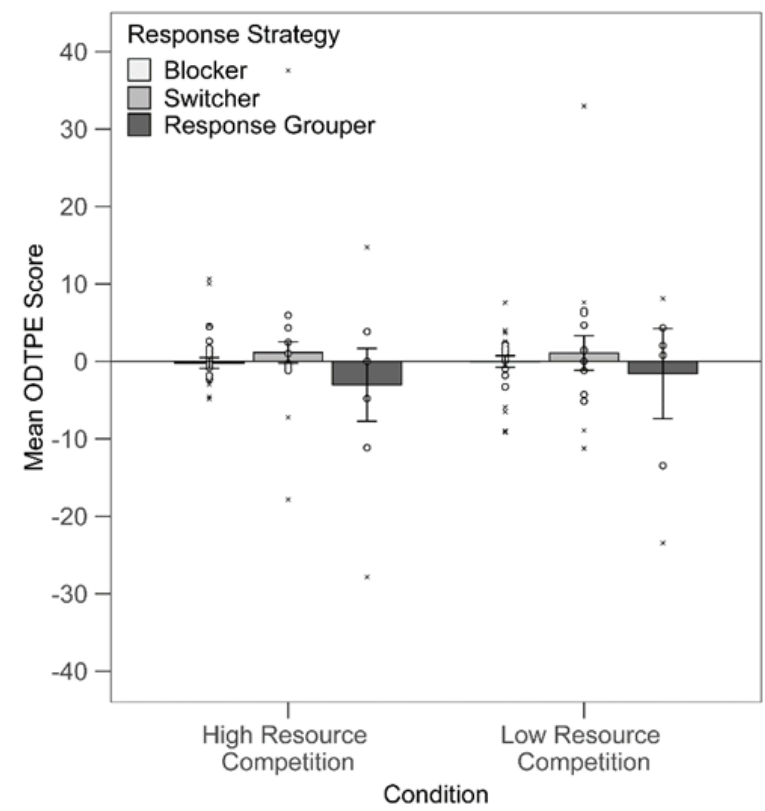

Fig. 4 Mean overall dual-task performance efficiency (ODTPE) scores achieved by the three strategy groups under both conditions of resource competition. Error bars represent \pm one standard error. Circles represent included and crosses excluded individual data after trimmed mean ( $20 \%$ criterion) As becomes evident from the figure, the pattern of effects was very similar under both conditions of resource competition. Independent of high or low competition for perceptualcognitive resources in the processing code dimension, the switcher achieved the highest multitasking efficiency of all groups. Their positive ODTPE score even reflects a small 
multitasking benefit compared to the task throughput in single-task conditions. In contrast, the blocker's efficiency fluctuated around zero, indicating that they were approximately as efficient in the dual-task as in single-task blocks. Only the response grouper showed clear multitasking costs, that is, they were less efficient in both conditions of resource competition when working on the two tasks concurrently compared to single-task performance.

Comparing the absolute efficiencies of the different strategies across the different conditions, only the efficiency of the switching strategy seemed to increase with decreasing degree of resource competition. However, all in all, the differences in multitasking efficiency achieved with the three different sorts of response organization remained relatively small, even in the condition of low resource competition. In order to compare the multitasking efficiency achieved by the three groups, their mean ODTPE scores were analyzed using a robust implementation of a heteroscedastic mixed 3(preferred strategy) x 2(resource competition) ANOVA. Because the subgroups varied considerably in their size, and the Levene test for both conditions (low resource competition: $F(2,38)=4.94, p=.012$; high resource competition: $F(2,38)=5.71, p=.007)$, as well as the Box test, $F(6,1964.89)=6.16, p<$ .001 , were significant, a robust ANOVA based on trimmed means $\left(M_{t}\right)$, as suggested by Mair and Wilcox (2019), was used. Neither the main effect of response strategy, $F(2,36.72)=1.29$, $p=.287, \varepsilon^{2}=.23$, nor the main effect of resource competition, $F(1,24.29)=0.08, p=.779$, $\varepsilon^{2}=.10$, or their interaction, $F(2,36.72)=0.01, p=.992, \varepsilon^{2}=.21$, were statistically significant in this analysis.

Thus, even though an expected improvement in multitasking efficiency could be observed at least for the switching strategy with reduced resource competition, the effect was too small to generate a statistically significant interaction effect in the overall ANOVA. Nevertheless, we were interested in understanding the possible basis of this descriptive improvement in the switcher group and therefore explored for more subtle differences. Our subsequent tests were grounded in the rationale that multitasking benefits of overlapping 
processing, reflected in time gains, might particularly be realized when switching between tasks, specifically indicated by strikingly short switch times (see for a detailed description of this rationale, Brüning \& Manzey, 2018). To the extent that better multitasking efficiency of switchers in the low-resource-competition condition was due to facilitated overlapping processing, this should be reflected in higher (fast) switch rates than in the high-resourcecompetition condition. Accordingly, we contrasted the mean switch rates and specifically the rates of fast switches in the dual tasks with high versus low resource competition. Because the assumption of normal distributed paired differences underlying the paired t-test was violated in this group, a Wilcoxon signed-rank test was used for comparing their switch rates between the two conditions. The test indeed revealed a significant difference of the switch rate in switcher, $Z=-2.48, p=.010$, with a somewhat lower switch rate in the condition of high $(M=$ $51.7 \%$, standard error, $S E=7.8)$ compared to low $(M=57.3 \%, S E=7.4)$ resource competition. In addition to this higher switch rate, also the average absolute number of fast switches increased from high $(M=275, S E=62)$ to low $(M=368, S E=79)$ resource competition, indicating a significant increase in incidences of overlapping processing, $t(12)=$ $2.83, p=.015, d=0.7$

\section{Discussion}

The basic finding of three groups, differing in their preference for a blocking, switching or response grouping strategy when performing two tasks concurrently in a selforganized manner, replicates the results of previous research (Brüning et al., 2020; Damos \& Wickens, 1980; Reissland \& Manzey, 2016). The three strategies were performed consistently and again occurred spontaneously, that is, independent of any instructions other than to optimize dual-tasking performance. This finding provides further converging evidence for the robustness of these individual differences. Furthermore, also the fact that all three ways of response organization led to a comparable multitasking efficiency when the two tasks 
competed for essentially the same perceptual, central processing and response-related resources (Wickens, 2002), was expected and confirms results of our previous research (Brüning et al., 2020; Reissland \& Manzey, 2016, Exp. 2).

However, unexpectedly, there were no significant differences between the conditions of high and low between-task resource competition at the level of the whole sample, neither in the multitasking efficiency nor in the switch rate. At the level of the individual response strategies, also contrary to our expectations, neither the choice nor the efficiency of response strategies was significantly affected when the competition for processing-code resources was reduced. This was evident in the dual tasks where a spatial and a verbal task had to be performed concurrently. Although these tasks required different processing codes (i.e., different pathways of processing), individuals applying one of the interleaving strategies did not significantly benefit from the presumably lower risk of dual-task interference compared to blocker. At a descriptive level, individuals preferring a switching strategy just showed a tendency to intensify their strategy by increasing their switch rate associated with a significantly higher degree of overlapping processing in the condition with lower resource competition. This was in line with our expectations that switcher would try to make more use of opportunities for overlapping processing when the competition for same resources was lower. However, the resulting improvement in multitasking efficiency remained only small and was insufficient to become statistically significant compared to the efficiency of blocker even under these conditions. Furthermore, response grouping, representing the other taskinterleaving strategy, always yielded the worst efficiency, regardless of whether the two tasks competed for the same processing-code resources or not.

The stability of the response strategies and the lack of statistical significance of the differences in multitasking efficiency between the groups for the condition of reduced resource-competition contrasts previous reports of better dual-task performance due to less dual-task interference when the processing codes of two tasks were separated (Herdman \& 
Friedman, 1985; Wickens et al., 1983; Wickens \& Liu, 1988; Wickens \& Sandry, 1982).

However, in this previous research, the separation of verbal versus spatial processing codes often was not limited to just the perceptual-cognitive resources, as in our first experiment, but also extended to a separation of response-modalities in terms of manual versus vocal responding (Göthe et al., 2016; Schaeffner et al., 2018; Tsang, 2006; Wickens et al., 1983; Wickens \& Liu, 1988; Wickens \& Sandry, 1982). This raises the question whether our null effects concerning differences in the response strategies' efficiency occurred because both tasks, although separated with respect to verbally and spatially coded perceptual-cognitive resources, still competed for similarly coded response-related resources (i.e., manual responding).

The critical role of a separation of response-related resources for multitasking efficiency has specifically been revealed in recent research by Göthe et al. (2016). Moreover, their research suggests, that there are specific compatibility relations between central processing codes (verbal, spatial) and response-modalities (vocal, manual), which are important in this respect. Using the PRP paradigm, the authors showed that the degree of interference between tasks, and thus the dual-tasking costs, depends to a large extent on the specific mapping of what they refer to as the stimulus feature and the response feature/modality of the tasks to be performed ${ }^{2}$. More precisely, dual-tasks combining a task relying on verbal-vocal stimulus and responses features with a task relying on spatial-manual stimulus and responses features resulted in lower dual-task costs than the reversed mapping. Göthe et al. (2016) explained their finding with the common coding principle in the framework of the theory of event coding (Hommel et al., 2001; Prinz, 1990). They assume that more similar features of stimuli and responses (e.g., verbal vs. spatial representation) facilitate correct bindings between stimuli and responses within one task and reduce risks of

\footnotetext{
${ }^{2}$ Note that basically the terms "stimulus and response features" used by Göthe et al. (2016) refer to similar if not identical concepts as the distinction of spatial versus verbal processing resources in the MRT (Wickens, 2002).
} 
crosstalk across tasks. Such mechanism might also explain earlier findings in human factors research pointing to the fact that multitasking might not only benefit from a separation of processing-code resources per se but specific compatibility relations between the different resources (e.g., Wickens et al., 1983; Wickens \& Liu, 1988). In addition, the fact that both tasks in our research involved manual responding may also have led to some additional structural task-interference effects resulting from activating and executing responses with both hands almost simultaneously (Heuer, 1990; Spijkers \& Heuer, 1995, 2004). These detrimental effects could have masked any possible benefits due to the separation of central processing resources. This would explain the other striking result of our first experiment, namely that response-grouping was the least efficient strategy with considerable multitasking costs even under conditions of low resource competition.

Based on these considerations, we conducted a second experiment in which we systematically varied the degree of resource competition for processing codes on both, the stages of perceptual-cognitive, and response-related processing according to MRT (Wickens, 1984, 2002). In addition to testing our general hypothesis about the importance of resource competition for the efficiency of different strategies of response organization, this approach also allowed to disentangle the relative effects of separating processing code resources for perceptual-cognitive processing and/or response-related processing or specific combinations of these resources on the use and efficiency of these strategies.

\section{Experiment 2}

The second experiment expanded the separation of processing code resources to also include variations of whether two concurrent tasks compete for the same or different response-related resources in terms of manual versus vocal responding. Altogether, four different dual-task conditions were realized in this experiment, covering a continuum from maximal to minimal resource competition. In the first condition (maximal resource 
competition) two tasks had to be performed concurrently, which competed for the same resources with respect to input modality (visual), processing code for perceptual-cognitive processing (spatial) and output modality (manual). The second condition included tasks, which were different with respect to demands of processing-code resources needed for perceptual-cognitive processing (verbal vs. spatial), but competed for the same processing code for responding (manual responding) and the same input modality (visual). The third condition involved the reverse with respect to processing-code separation, meaning that the response-related resources were coded differently (i.e., manual vs. vocal responses), whereas the perceptual-cognitive resources relied on the same code (verbal) and input modality (visual). Lastly, in the fourth condition the resource competition was kept minimal, with both tasks just competing for same visual resources, but being completely separated with respect to the processing codes for perceptual-cognitive processing (verbal vs. spatial) and responding (manual vs. vocal). The first and second condition corresponded to two dual-task conditions of Experiment 1. The third and fourth condition, were newly introduced in Experiment 2 to investigate the (additional) effects of a separation of response-related resources.

The main focus of Experiment 2 remained on how the efficiency of individually preferred strategies of response organization would be affected by the extent of resource competition between tasks. However, an even more basic question is where these individual differences in the preference for specific response strategies originate from. This has not yet been investigated in previous research. As a first attempt to address this question, we further included two measures assessing individual differences, which we assumed to be related to the strategies of response organization. In this regard, we assumed that individual differences in the individual working memory capacity (WMC) could play a role because it has been shown to affect different aspects of executive functions and attentional control (Engle, 2002; Engle \& Kane, 2004; Kane et al., 2007) and the preference for overlapping processing (Brüning \& Manzey, 2018). Accordingly, we expected that a higher WMC might be also 
associated with a higher extent of interleaving two tasks. In addition, we assumed that the response strategies found might be associated with the individual's polychronicity (Slocombe \& Bluedorn, 1999). Polychronicity is a construct that reflects “[...] a combination of past experience with multitasking and a stable tendency to perceive multitasking as enjoyable and rewarding rather than stressful [...]" (Poposki \& Oswald, 2010). As such it represents a measure for evaluating an individual's attitude towards multitasking in real-life situations. We expected that a more positive attitude towards multitasking would be reflected in a higher number of switches between two tasks.

\section{Method}

Since the paradigm and the stimuli for both experiments were identical and the procedure and data analysis for both experiments were largely similar, only deviations from Experiment 1 are reported for this experiment.

\section{Participants}

A total of 50 volunteers took part. The dataset of three participants were excluded from the analysis. One of them was rejected because of technical problems in recording vocal replies, one because of high error rates (ER >20\%), and one because of not complying with the instruction to perform both tasks of a dual task with equal priority. The final sample included 47 participants (22 female, mean age $=26$ years, $S D=3.2$ years, range $=19-33$ years) all of which met the requirements and were reimbursed as in the first experiment.

\section{Tasks}

The same spatial memory-search task, spatial classification task and verbal classification task as in Experiment 1 were used. However, the verbal memory-search task was changed with respect to its response modality. Instead of responding to the task by pressing different keys on the keyboard as in Experiment 1, the participants were required to say either "gruen" (German for "green") in case the presented probe letter pair matched one of 
those target letter pairs they remembered, or "rot" (German for "red") if not. All other tasks were responded to as in Experiment 1. Out of the four tasks, a total of four dual-task combinations were composed differing in their competition for processing-code resources as described in Figure 5.

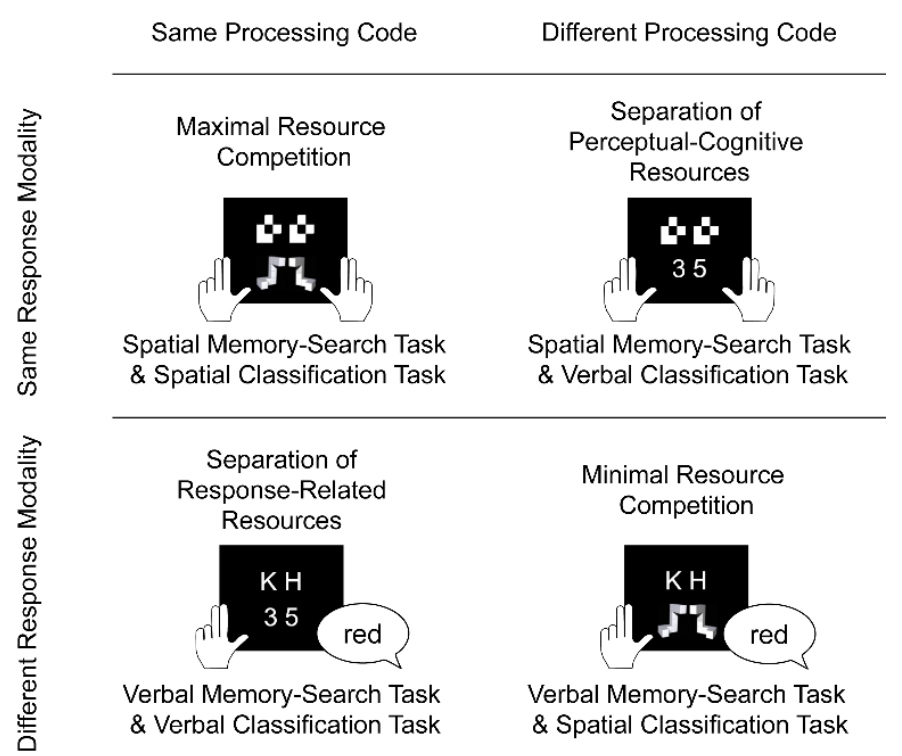

Fig. 5 Example of the four defined dual-task conditions. Each dual task comprised a classificationand a memory-search task. In the maximal resource competition, the processing-code (spatial), as well as the response modality, was the same (manual). Conversely, in the minimal resource competition both, the processing-code (verbal and spatial) and the response modality (manual and vocal) were separated. In the other two conditions, either the processing-code (separation of perceptual-cognitive processing) or the response modality (separation of response-related processing codes) was separated

The condition of maximal resource competition comprised the spatial classification and the spatial memory-search tasks, involving spatial stimuli and manual responses, respectively. The condition of separated processing codes needed for perceptual-cognitive processing included the verbal classification task and the spatial memory-search task, involving different stimuli (i.e., spatial and verbal) for both tasks but same processing code for responding (manual). The corresponding condition with separation of processing codes for responding comprised the verbal classification task and the verbal memory-search task, both involving verbal stimuli, but different codes of responding (manual vs. vocal). Finally, the 
condition of minimal resource competition comprised the verbal memory-search and the spatial classification tasks, demanding different processing-code resources for perceptualcognitive processing (verbal vs. spatial) as well as responding (manual vs. vocal). Here, additional care was taken to use particularly compatible stimulus-response feature bindings for the two component tasks (verbal-vocal; spatial-manual) to separate the processing-code resources in a supposedly optimal way (Göthe et al., 2016; Wickens et al., 1983). Thus, overall, we obtained one dual-task condition with maximal resource competition, two dualtask conditions with medium resource competition and one with minimal resource competition, the latter also optimized with respect to resource compatibility considerations.

\section{Apparatus}

The response recording was controlled by a custom-made JAVA software and a Python (Version 3.6.4) based application running on the same PC workstations as in Experiment 1. At each workstation, the red and green color points applied on the predefined keys of the standard keyboards were replaced by white and black points to prevent interference with the vocal responses in the verbal memorization task. For the vocal responses, answers were recorded using a headset with an integrated microphone (Corsair Void Pro) and a sampling rate of $44100 \mathrm{~Hz}$. The individual threshold was defined in the beginning of the experiment for each participant and served as a response threshold (i.e., the response time corresponded to the voice onset). Whenever this threshold was reached the response recording started and ended when the signal fell below the threshold again. The next stimulus was presented to the participant automatically when the record of the response started, thus, there was also no response-stimulus interval in the verbal memory-search task. After recording, the verbal answers were sent to and classified by google Cloud Speech-toText. The connection between the custom-made JAVA software and the google Cloud Speech-to-Text was established via a custom-made Python application using the following 
libraries: pypiwin32, 223; PyAudio, 0.2.11; google-api-python-client, 1.7.7;

SpeechRecognition, 3.8.1. Note that google did not store any data at any time.

\section{Additional Measures}

Working Memory Capacity. The individual WMC of each participant was assessed by the automated operation span task (Ospan; Turner \& Engle, 1989; Unsworth et al., 2005). In this task the participants solve a series of simple arithmetical operations while trying to remember a number of letters (set sizes 3-7). One letter was presented after each math problem. The letters should then be recalled at the end of each test trial. The Ospan was presented on the same screen as the dual tasks using the Psychology Experiment Building Language (Mueller, 2012; Mueller \& Piper, 2014). The Ospan had a Cronbach's alpha reliability coefficient of .78 and a test-retest reliability of $r=.83$.

Polychronicity. The degree to which an individual is polychronic was assessed with the Multitasking Preference Inventory (MPI; Poposki \& Oswald, 2010). This questionnaire contains statements that describe a person's personal preferences for performing multiple tasks in their daily life (e.g., "I prefer to work on several projects in a day, rather than completing one project and then switching to another"). The MPI comprised 14 items based on a five-point Likert scale and the entire scale had a Cronbach's alpha reliability coefficient of .88 and a test-retest reliability of $r=.83$.

\section{Procedure}

The experiment included three sessions, comparable to the first experiment. The first session lasted about 1.5 hours and was dedicated to an assessment of working memory capacity via the Ospan task, followed by a familiarization and practice of the different single and dual tasks. The second and third session lasted one hour each and included the actual data collection of the experiment with the four dual tasks. In order to have one dual task with different response modes (manual, vocal) included in each session, the dual tasks with maximal response competition and with separated response-related resources were always 
performed in the same session, as were the dual tasks with separated perceptual-cognitive resources and minimal resource competition. The sequence of sessions including these conditions was balanced across participants. The polychronicity questionnaire was always filled in after the last experimental block of the third session.

Always, one to three volunteers were tested simultaneously at independent PC workstations, separated by opaque screens. While performing the single and dual tasks, the participants wore earplugs and received white noise with 50db to ensure that they would not be distracted by the other participants' vocal responses. All other procedural details concerning the general structure of the three experimental sessions and the presentation of tasks corresponded to the procedure of the first experiment, with only one exception concerning the provision of performance feedback for the single and dual tasks with vocal responses. Principally, the online classification of vocal responses for the verbal memorysearch task allowed feedback to be given to the participants in the same way as for the other tasks. However, for the few answers for which the online classification could not be performed correctly, e.g., due to inaccurate pronunciation, randomly selected responses were implemented in the feedback. Note that this approach did not affect subsequent analyses of the data. Because it only applied to few cases, a distortion of the percentage of correctly answered trials was hardly noticeable. After the experiment, the participants stated that they did not notice any difference between the feedback for the tasks to be answered manually and the estimated feedback for the task that had to be answered vocally.

\section{Design}

The experiment entailed a 2 (perceptual-cognitive processing codes: verbal vs. spatial) x 2 (response-related processing code: vocal vs. manual) x 3 (preferred strategy: blocker vs. switcher vs. response grouper) experimental design. The first two factors represented withinsubject factors, whereas the factor preferred strategy represented a between-subject factor. Note, that the latter factor again resulted from the post-hoc categorization of the individual 
strategies of response organization observed in the different dual-task conditions. It was included for the main analyses addressing the impact of different multitasking strategies on multitasking efficiency.

\section{Data Analyses}

All aspects of data analyses concerning dual-task performance, that is, the definition of different trial types considered, the identification and reliability analyses of individual strategies of response organization in the different dual-task conditions, and the multitasking efficiency achieved with the different strategies in the different conditions, corresponded to the approach used in the first experiment.

The classification of the Google Cloud Speech-to-Text recognition failed in $8.13 \%$ of the vocal responses. The failures were classified manually by listening. For $0.45 \%$ single-task trials and $0.75 \%$ dual-task trials of the vocal responses no classification could be obtained (e.g., recorded breathing), thus, they were handled like response errors. For further analyses, data of each participant were collapsed per trial type, across tasks and experimental runs, but separately for the four different dual-tasks. This yielded to 202 single task trials $(S D=42)$ and 412 dual-task trials $(S D=78)$ per task, dual-task condition and participant on average. Outliers were identified as described in Experiment 1 resulting in an average of $4.44 \%$ of single task trials $(S D=1.34 \%)$ and $8.5 \%$ of dual-task trials $(S D=7.16 \%)$ per participant.

On the resulting data, we performed three sets of analyses. The first one included an analysis of the multitasking efficiency at the group level according to the 2(perceptualcognitive processing code) $\mathrm{x}$ 2(response-related processing code) design. It mainly served to evaluate overall effects of resource separation. The second one addressed potential shifts in the choice of response strategies across the conditions. Because no significant shifts were expected for the transition from the condition of minimal to the conditions of medium resource competitions, potential shifts in the choice of strategies were statistically compared only for the two extreme conditions of resource competition (maximal vs. minimal). The third 
and most important set of analyses addressed the multitasking efficiency of different ways of response organization in the different dual-task conditions. These analyses involved comparisons of the three strategies within each condition, as well as a comparison of the efficiency of the two task-interleaving strategies across the different conditions. The latter analysis included only participants using the same strategy in all conditions.

The data analysis addressing to what extent the preferences for interleaving versus blocking response strategies were related to the individual WMC and degree of polychronicity were tested by correlating the participants' mean switch rate in each dual-task condition with both, their partial scores achieved in the Ospan (Conway et al., 2005; Friedman \& Miyake, 2005; Redick et al., 2012), as well as their mean score achieved in the MPI (Poposki \& Oswald, 2010). Note that these correlations were calculated across all participants, independent of what strategy of response organization they applied, thus, using the natural variation of switch rates between these strategies as a basis.

\section{Results}

\section{General Effect of Lowered Resource Competition}

At the level of the whole sample, the error rates again were generally low with all single-task and dual-task error rates $<6 \%$ (see Supplemental Material 1 for more details on IRIs and ERs). Besides, the participants complied well with the instruction of equal task priority $($ Mean $=1.03, S D=0.15)$. In contrast to the first experiment, now considerable differences in mean switching rates and mean ODTPE scores between different resource competition conditions emerged (Table 2).

Table 2. Mean percentage and standard deviation (SD) of switch rate and ODTPE scores per condition of resource competition.

\begin{tabular}{lll}
\hline Condition of resource competition & Switch Rate (SD) & ODTPE (SD) \\
\hline Maximal Resource Competition & $40.23(38.02)$ & $-0.01(9.81)$ \\
Perceptual-Cognitive Separation & $42.58(37.59)$ & $1.62(8.82)$
\end{tabular}


Response-Related Separation

$49.95(35.48)$

$7.11(10.71)$

Minimal Resource Competition

As becomes evident, mean switch rates and ODTPE scores increased specifically in the two conditions where response-modalities were separated, reflected in main effects of response-related processing code in the 2 (perceptual-cognitive processing code) x 2 (response-related processing code) ANOVA, switch rate: $F(1,46)=10.87, p=.002, \eta_{\mathrm{G}}{ }^{2}=.01$, ODTPE score: $F(1,46)=32.25, p<.001, \eta_{\mathrm{G}}{ }^{2}=.11$. All other main effects and interactions for both dependent variables were non-significant (all $p>.148$ ).

\section{Reliability and Flexibility of Individual Multitasking Strategies}

The split-half reliability assessments revealed that again all participants but one could be reliably classified concerning their preferred strategy of response organization in the different dual-task conditions (Figure 6). For our further statistical analyses, however, the data of the deviating individual was retained according to the classification based on the whole data set because the slight inconsistency only concerned one of the four dual-task conditions and only ranged within the task-interleaving strategies. As also the mean error rates of the three strategy subgroups were $<6 \%$ in all four conditions they were not considered in the further analyses (see also Supplemental Material 1 for more details on IRIs and ERs).

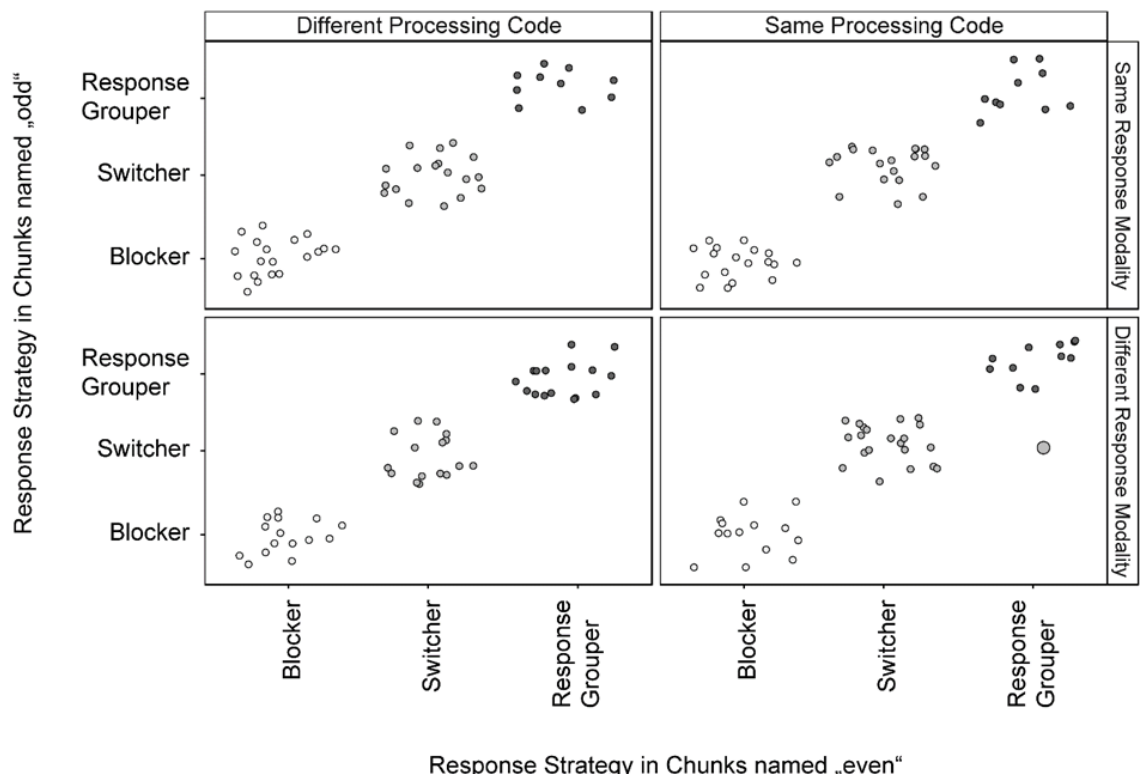


Fig. 6 Results of the split-half reliability assessments. Circles represent the classified response strategy for each individual based on aggregated data in "odd" versus "even" labeled chunks in the four different dual-task conditions. Data points are jittered for reasons of illustration

The number of blocker, switcher and response grouper identified in each of the four conditions of resource competition and incidents of shifts between the response strategies across the four conditions are summarized in Figure 7.

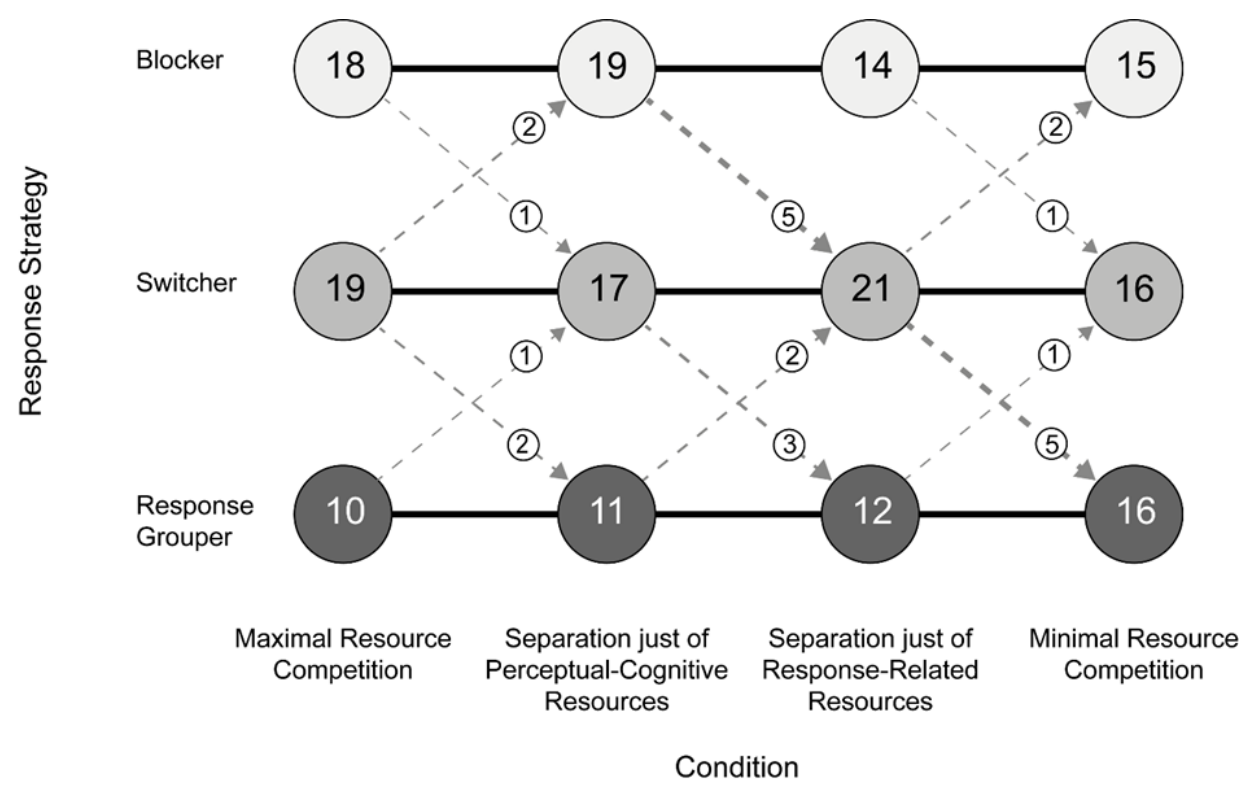

Fig. 7 Number of participants using the blocking, switching or response grouping strategy of response organization in the different dual-tasks (large circles). Small circles represent the number of participants who shifted between strategies across dual-task conditions. The thickness of broken lines indicating shifts corresponds to the relative number of participants changing their strategy in a given direction. Note that no shifts occurred between blocking and response grouping

As becomes evident from the figure, most of the participants used their preferred strategy of response organization in a very consistent way, independent of the degree of resource competition. An inspection of the individual data revealed, that actually about two thirds of the participants (30 out of 47) used the same strategy in all four dual-task conditions.

The majority $(n=10)$ of the other 17 participants just changed between the two strategies of task interleaving, i.e., preferred to switch in some conditions and to group their responses in others. Of these ten people, seven gradually shifted from switching to response 
grouping under conditions of decreasing resource competition, while the other three just shifted once between switching and response grouping. Only seven participants were found who changed between blocking and switching. However, only three of them did so in a systematic way, obviously responding to the separation of processing-code resources. They preferred to block the tasks in the condition with maximal resource competition but changed to a switching strategy when the resource competition between the two tasks was reduced. The other four participants (two blockers and two switchers) just shifted once to another strategy. However, because they always used the same strategy in the two extreme conditions with maximal and minimal resource competition, these changes did not seem to be related to the degree of resource competition in any systematic manner.

In sum, the largest difference in the distribution of response strategies was evident when comparing the two extreme conditions of maximal versus minimal resource competition, confirmed by a significant McNemar's Chi square test, $\chi^{2}(2, \mathrm{n}=47)=7.5, p=$ .024. However, most of these shifts resulted from individuals preferring to switch under maximal resource competition but to group their responses under minimal resource competition. That is, they just shifted between the different ways of interleaving the two tasks. In contrast, the vast majority of blocker preferred to keep both tasks as separated as possible, even in the condition of minimal resource competition, and none of the switcher or response grouper ever shifted to a blocking strategy when the resource competition was minimal.

\section{Efficiency of Individual Multitasking Strategies}

An overview about the multitasking efficiency achieved in the different dual-task conditions by applying different strategies of response organization is illustrated in Figure 8 . Displayed are the mean ODTPE scores with scores above zero reflecting a higher task throughput under dual-task compared to single-task conditions and scores below zero indicating multitasking cost effects. Because one third of the participants shifted their preferred strategies between conditions at least in one condition, the efficiency differences 
between all individuals could not be compared directly across conditions within one analysis (i.e., testing the interaction would have been based on dependent measurements). Thus, instead of analyzing the data according to the $2 \times 2 \times 3$ design, which would have been the most elegant approach if the post-hoc classification had led to three completely independent "strategy" groups across all dual-task conditions, we had to split the first set of analyses and compare the mean multitasking efficiency of blocker, switcher and response grouper within each of the four resource conditions separately. Because the comparable conditions of maximal resource competition and separated perceptual-cognitive resources of the first experiment did not show significant changes dependent on the preferred response strategies, we did not expect to see differences between the strategies in these conditions in the second experiment, either. Hence, only the two conditions of separated response-related resources and minimal resource competition were considered for Bonferroni-Holm adjustments of local alpha levels.

This first set of analyses was then complemented by an intraindividual comparison of the characteristics and efficiency of strategies of those participants, who showed a stable preference for one of the two task-interleaving strategies in the two extreme conditions of minimal and maximal resource competition.

Comparison of efficiency between strategies of response organization. The multitasking efficiency achieved by the three types of response organization was compared separately for each of the four different dual-task conditions. As in Experiment 1, robust implementations of a heteroscedastic one-way ANOVA based on trimmed means (Mair \& Wilcox, 2019) were used. Pairwise post-hoc comparisons of the trimmed means were made using Fisher's Least Significant Difference Test (LSD, Howell, 2016; lincon function of the R-package WRS2). 


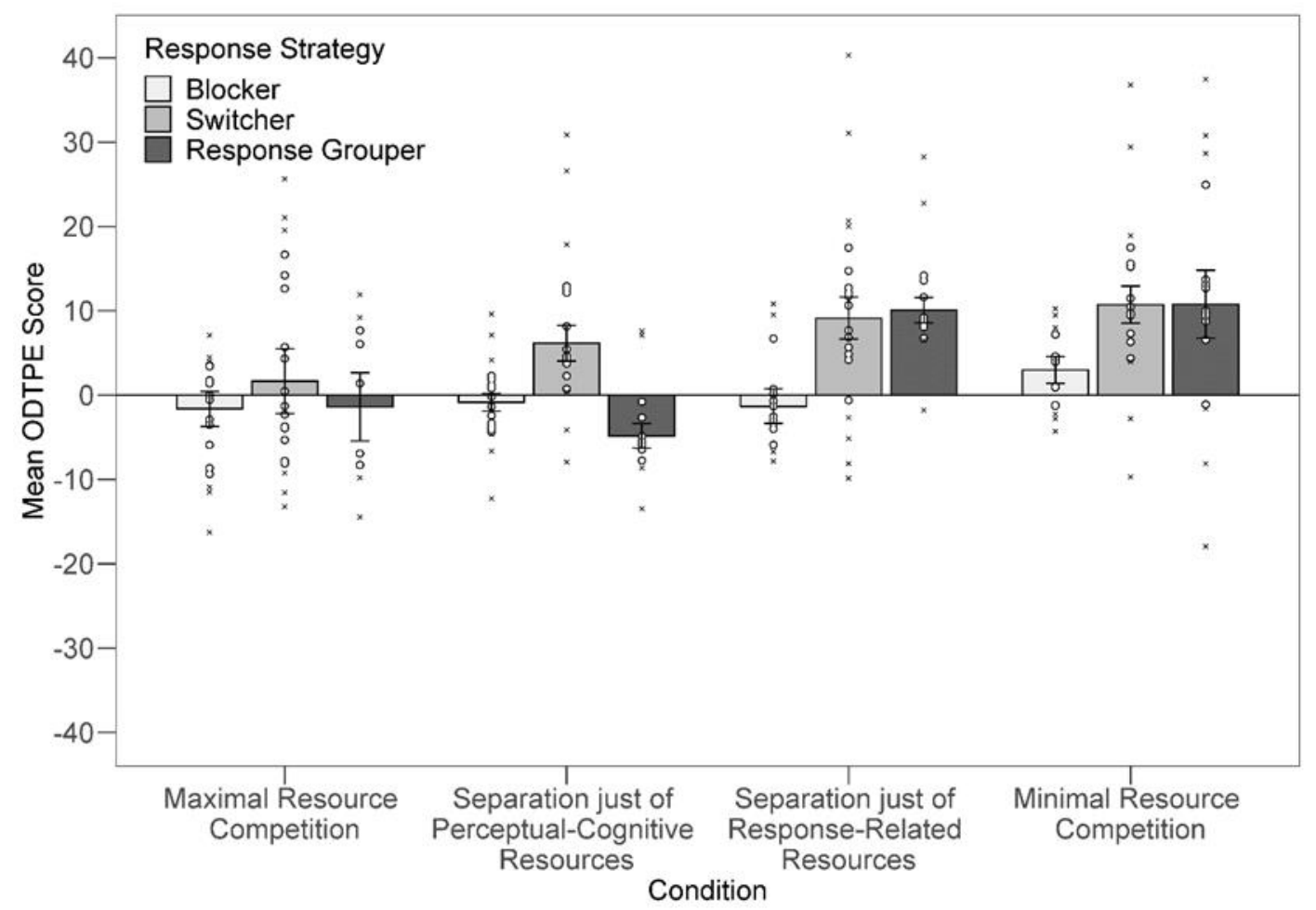

Fig. 8 Mean overall dual-task performance efficiency (ODTPE) scores of each response strategy group in the four different conditions of resource competition. Error bars represent \pm one standard error.

Circles/crosses represent individual data included/excluded in the calculation of trimmed means (20\% criterion)

As expected and in line with results from the first experiment, the differences in mean ODTPE scores between different strategies of response organization were only low and not statistically significant when the two tasks to be performed concurrently essentially demanded the same resources, $F(2,12.52)=0.34, p=.715, \varepsilon^{2}=.18$.

However, this pattern changed already in the condition where the codes needed for perceptual-cognitive processing of the two tasks were separated. Participants using the switching strategy now achieved a clear multitasking benefit, whereas the other strategies still led to more or less pronounced cost effects compared to single-task performance. A similar, albeit non-significant trend was observed in comparable dual-task conditions in the first experiment. Yet, this time it led to a significant main effect, $F(2,15)=9.73, p=.002, \varepsilon^{2}=$ 73. Post hoc pairwise comparisons revealed that switcher $\left(M_{t}=6.2 \%, S E=2.1\right)$ performed 
significantly better than both, blocker $\left(M_{t}=-0.9 \%, S E=1.1 ; p=.007\right)$ and response grouper $\left(M_{t}=-4.8 \%, S E=1.5 ; p<.001\right)$, whereas response grouper performed even significantly worse than blocker $(p=.037)$.

An even more pronounced change of the overall pattern of effects occurred in the condition where the resources needed for response-related processes got separated. Now, also response grouping was efficient, and participants using either of the two task-interleaving strategies achieved considerable multitasking benefits, whereas blocker still produced small multitasking costs, $F(2,18.64)=13.46, p<.001, \varepsilon^{2}=.66$. Post-hoc comparisons confirmed that in this condition both, switcher $\left(M_{t}=9.1 \%, S E=2.5\right)$ and response grouper $\left(M_{t}=10.1 \%\right.$; $S E=1.5)$, achieved a higher multitasking efficiency than blocker $\left(M_{t}=-1.3 \% ; S E=2.1\right.$; both $p<.002)$, whereas no significant differences emerged between the two task-interleaving strategies.

Essentially the same pattern of effects also emerged in the condition of minimal resource competition where again clear differences between blocker and the two other subgroups emerged, $F(2,16.33)=4.6, p=.026, \varepsilon^{2}=.49$. In this condition even blocker showed slight multitasking benefits $\left(M_{t}=3 \%, S E=1.6\right)$. However, they were still outperformed by switcher $\left(M_{t}=10.7 \%, S E=2.2 ; p=.011\right)$ and response grouper $\left(M_{t}=\right.$ $10.8 \%, S E=4 ; p=.094)$, although the difference between blocker and response grouper failed to reach the usual level of statistical significance, presumably due to a relatively high standard error in the latter group.

\section{Intraindividual comparison of task-interleaving strategies between conditions of}

maximal and minimal resource competition. The pattern of effects shown in Figure 8 suggests that specifically the efficiency of the switching and response grouping strategies benefited considerably from a separation of response-related processing-code resources. In order to better understand the underlying determinants of these efficiency gains, we complemented the foregoing analyses by an intraindividual comparison of the efficiency, 
characteristics and related time-gains of the switching and response grouping strategy across conditions. In order to prevent a significant reduction in statistical power (i.e., by excluding too many participants), only the two most extreme conditions of maximal and minimal resource competition were included in these analyses.

Overall, twelve participants used a switching strategy in the two conditions. With this strategy, they were able to increase their multitasking efficiency from a mean ODTPE score of $3.2 \%(S E=3.6)$ in the condition with maximal resource competition to $13.1 \%(S E=3.2)$ in the condition with minimal resource competition, $t(11)=3.2, p=.008, d=0.92$. A detailed analysis of how they applied their switching strategy revealed that they increased their mean switch rate from $44.2 \%(S E=7.8)$ in the condition with maximal resource competition to $60.6 \%(S E=3.9)$ in the condition where the processing-code resources were maximally separated, $t(11)=-2.53, p=.028, d=.73$. This was accompanied by a slight, albeit not significant increase of the mean number of fast switches indicating increased overlapping processing in this subgroup, from $297(S E=86)$ to $344(S E=29)$ in the conditions of maximal and minimal resource competition, respectively. Even more important, also the efficiency of overlapping processing in this group increased with decreasing resource competition. This was reflected in the mean IRI for fast switches which was 253,6 ms faster than the average single-task IRI in the condition with maximal resource competition but even $701,8 \mathrm{~ms}$ faster than the average single-task response times in the condition with minimal resource competition. Overall, this effect contributed to a general acceleration of responses in switch trials compared to the respective single-task responses (i.e., an increased mean timegain or switch benefit) from $65 \mathrm{~ms}(S E=36)$ under maximal resource competition to $436 \mathrm{~ms}$ $(S E=100)$ under minimal resource competition, $t(11)=3.74, p=.003, d=1.08$.

A similar analysis was conducted for the nine response grouper who did not change their strategy across the two extreme conditions. They were able to increase their overall multitasking efficiency from a mean ODTPE score of $-2.3 \%(S E=3.1)$, reflecting some 
multitasking costs in the condition of maximal resource competition, to a clear multitasking benefit $(\mathrm{M}=9.7 \%, \mathrm{SE}=4.4)$ in the condition with minimal resource competition, $t(8)=2.32$, $p=.049, d=0.77$. These participants performed the two tasks A and B most of the time in an alternating $\mathrm{ABABAB}$ order with grouped responses. Accordingly, they performed the tasks virtually pairwise with first selecting the two responses to the tasks and then executing these responses in close succession. In order to analyze the time gains achieved with this particular strategy in the two dual-task conditions compared to the corresponding single-task baselines, a different approach had to be pursued than for the switcher. For response grouper, we contrasted the mean time needed to complete an $\mathrm{AB}$ task pair under dual-task conditions when grouping the responses with the time needed for the two tasks under single-task conditions as assessed by the sum of mean single-task IRIs for tasks A and B. This analysis revealed that whereas the response grouping strategy was associated with time losses compared to single-task performance of about $165 \mathrm{~ms}(S E=69)$ in the condition of maximal resource competition, a clear time gain of on average $95 \mathrm{~ms}(S E=54)$ was achieved with each task pair in the condition of minimal resource competition, $t(8)=2.65, p=.029, d=0.88$.

\section{Correlations between individually preferred strategies of response organization}

and additional measures of individual differences. For each dual-task condition, the individual participants' switch rates were correlated (Pearson) with both, their partial Ospan score and their sum score in the MPI. All participants were considered in these analyses, independent of their strategy of response organization. For the Ospan task, no significant correlations were found in either condition, all $\mathrm{p}>$.792. In contrast, significant correlations emerged between the individuals' switch rate and the MPI in all conditions, when BonferroniHolm correction was considered: maximal resource competition, $r(45)=.447, p=.002$, separated perceptual-cognitive resources, $r(45)=.402, p=.005$, separated response-related resources, $r(45)=.382, p=.008$, and minimal resource competition, $r(45)=.338, p=.02$. 


\section{Discussion}

The second experiment was conducted to shed more light on how a separation of resource demands of two tasks, especially in terms of response-related processing codes according to MRT (Wickens, 2002), would affect the choice and efficiency of a certain type of response organization when performing the tasks concurrently.

First, the results provide further evidence for the spontaneous and reliable preference of individuals for a blocking, switching or response grouping strategy. In addition, they confirm the finding of the first experiment that the choice of response strategies seems to be largely independent of the similarity of the processing codes needed to process the two tasks. This is reflected in the fact that the vast majority of individuals (64\%) choose the same type of response strategy in all four conditions of resource competition. Moreover, most individuals (21\%) using a different response strategy for one or two of these conditions only shifted between the two interleaving strategies (i.e., from switching to response grouping with reduced resource competition). Hence, this shift between task-interleaving strategies seems to reflect a kind of intensification of task interleaving rather than a qualitative change. Overall, the results of Experiment 2 indicate that the choice of response strategy does indeed reflect stable inter-individual differences that are widely independent of the given characteristics of the tasks to be performed. The correlational analyses further suggest that these differences do not relate to differences in working memory capacity, but rather correspond to a distinction in the degree to which an individual favors a structured and rather predictable working environment.

Regarding the effect of lowered resource competition on multitasking efficiency, this time, a beneficial effect of separating response-related resources in particular was already evident on the basis of the whole sample data. However, a more differentiated picture emerged on the level of individual response strategies. The blocking strategy again turned out to be a rather conservative strategy, which neither led to clear benefits nor costs of 
multitasking in any of the different conditions. This is not surprising as individuals using a blocking strategy inherently avoid more frequent switches and the related risk of switch costs, but at the same time deprive themselves of the opportunity to save time by responding faster in task switches.

In contrast, the efficiency of the two interleaving strategies increased significantly in the dual-task conditions involving reduced resource competition. For the switching strategy, performance gains emerged already in the condition where processing codes for perceptualcognitive processing were separated. This marks a contrast to the results of Experiment 1, where this separation alone only led to a descriptive increase in multitasking efficiency. In Experiment 2, however, the increase in efficiency achieved with the switching strategy was even more pronounced in the conditions of separate response-related resources and minimal resource competition. This marked effect was even more evident for response grouper, as they were not yet efficient with separated processing codes for perceptual-cognitive processing, but benefitted strongly when the response-related resources were separated. In the conditions of separated response-related resources and minimal resource competition, participants using either type of interleaving strategy achieved a multitasking benefit of about $10 \%$ reflected in a higher task throughput under dual-task conditions compared to single-task performance.

For both, the individuals preferring to switch, as well as for those who prefer to group their responses, the finding of improved multitasking efficiency was grounded in the degree to which they overlap the processing of the two tasks. This was revealed by the more finegrained analyses of IRIs for the switcher, showing that the separation of codes of processing not only led to an increase in switch rates but also to a descriptively higher number of fast switches associated with significantly higher mean time-gains achieved with these switches. For response grouper time losses in the period before execution of the two responses in the condition of maximal resource competition turned into time gains in the condition with minimal resource competition. This fits to the finding of Brüning et al. (2020) suggesting that 
specifically those individuals who show an individual preference for task-interleaving strategies in self-organized multitasking also show a spontaneous preference for overlapping processing in the TSWP paradigm. It also supports the basic assumption of MRT that a separation of resources demanded by tasks make a parallel processing of tasks easier (Wickens, 1984, 2002). Finally, it also fits to the specific predictions of the theory of event coding (Göthe et al., 2016; Hommel et al., 2001), given that the tasks in the condition with minimal resource competition were additionally optimized with respect to the compatibility of processing-code resource pairings. However, the fact that most of the efficiency gains were already achieved with just the separation of response-related resources suggests that the additional consideration of specific resource compatibility effects probably contributed to a lesser extent to the improvement of multitasking efficiency than the unlocking of structural interference effects by separating the response modalities (Heuer, 1990; Spijkers \& Heuer, 1995, 2004). We will return to this in the General Discussion.

\section{General Discussion}

In the present study, we examined whether the choice and efficiency of individually preferred strategies of response organization in self-organized multitasking (i.e., blocking, switching, response grouping) depend on the degree to which two tasks compete for the same processing codes needed for perceptual-cognitive processing (verbal vs. spatial) and/or response-related processes (manual vs. vocal). Based on previous results (Brüning et al., 2020; Reissland \& Manzey, 2016) it was assumed that in case of high competition for processing codes either of the three strategies would be as (non-)effective as the other. By contrast, in conditions with lowered resource competition which, according to MRT (Wickens, 2002), should facilitate to process and respond to tasks in parallel, clear advantages were expected to arise for the switching and response grouping strategy. Both of these latter strategies are characterized by a sort of task interleaving which in principle would provide 
options for overlapping task processing. Furthermore, previous research has shown that these strategies are indeed particularly preferred by individuals who also tend to process tasks in an overlapping instead of a serial manner if possible (Brüning et al., 2020). Accordingly, it was expected that individuals adapt their preferred strategy of response organization to the degree of resource competition between tasks. In addition, it was assumed that the multitasking efficiency of individuals preferring a switching or response grouping strategy is the better, the more the processing codes needed for perceptual-cognitive processing and responding were separated. The results of the two experiments clearly contrast the former hypothesis but provide some support for the latter.

\section{Stability of Strategies of Response Organization}

Let us first turn to the impact of resource competition on the way the responses to the two concurrent tasks were organized. Confirming the results of our previous research (Brüning et al., 2020; Reissland \& Manzey, 2016), we again found three subgroups using a blocking, switching or response grouping strategy, respectively, when performing the different dual tasks in the two experiments. This finding was further complemented by reliability assessments, which showed that almost all participants in both experiments consistently pursued their strategies. However, in contrast to our assumptions, the distribution of preferred response strategies across individuals remained remarkably stable across all dualtask conditions. This held particularly true for the first experiment where virtually all participants (95\%) used the same response strategy throughout the two dual-task conditions independent of the different degree of resource competition between tasks. However, also in the second experiment in which the degree of competition for processing codes was varied even across four conditions, stable preferences were found for at least the majority of participants (64\%). The few exceptions among the participants (21\%) primarily seemed to intensify their preferred style of task interleaving by shifting from switching to response grouping with reduced resource competition. In this sense, the few shifts between strategies 
across conditions resembled the increase in switch rates in voluntary task switching reported by Mittelstädt et al. (2018; 2019) or Fröber and Dreisbach (2017) as a result of stimulus availability or task context.

The finding of a relatively high individual persistence with respect to a blocking strategy versus task-interleaving strategies of response organization contrasts the adaptability at the level of internal task processing, which has been shown based on research with the TSWP or PRP paradigm (Brüning \& Manzey, 2018; Lehle \& Hübner, 2009). For example, in the study of Brüning and Manzey (2018), individuals who preferred an overlapping processing of two tasks, were able to change to a more serial mode of processing in case of elevated risk of cross-talk between the two tasks they had to perform. The observed stability on the level of response organization in the current study suggests that this sort of adaptability with respect to the mode of processing might not necessarily transfer to the way of response organization. This points to the fact that basic ways of response organization in multitasking represent relatively strong habits which are difficult to change.

Such an assumption would also fit to earlier observations reported by Damos et al. (1983). Based on fine-grained analyses, similar to the ones used in the present research, they distinguished between a simultaneous, an alternating, and a massed strategy of response organization in performing a memory and a classification task concurrently. The first two strategies obviously correspond to the task-interleaving strategies found in our experiments, whereas the massed strategy resembles what we refer to as blocking. Damos et al. (1983) then tried to train participants preferring a massed or alternating strategy to change to the simultaneous strategy. Participants who had to change from alternating to simultaneous responding, i.e., between the two interleaving strategies, achieved a similar multitasking efficiency as individuals preferring the simultaneous strategy. However, individuals originally preferring a massed strategy lost much of their efficiency when applying the new way of response organization. Corresponding to our results, shifting from a strategy of separating 
tasks to interleaving tasks, i.e., shifting contrary to the individual preferences was obviously much more difficult than shifting between two different task-interleaving strategies.

One reason for the obviously stable individual bias for either a blocking strategy or task-interleaving strategies in the different dual-task conditions might be assumed in differences in cognitive abilities. This notion is further supported by our previous finding of a link between WMC and preferences for overlapping task processing (Brüning \& Manzey, 2018). However, only relatively low correlations between the observed rates of task switches and WMC were found in the second experiment. In contrast, significant correlations with the polychronicity measure emerged. This suggests that the basic distinction between preferences for blocking versus interleaving strategies might rather reflect a preference for more structured versus flexible ways to cope with concurrent tasks. A differentiation that would fit with the distinction of persistence and flexibility as two styles of meta-cognitive control resulting from different learning experiences or cultural influences (Hommel, 2015).

\section{Efficiency of Strategies of Response Organization}

A second major question addressed in the present study concerned the efficiency of the different strategies of response organization. As expected, the results show that strategies of task interleaving are more efficient than a blocking strategy in dual-task situations where the two tasks compete for different rather than same processing-code resources. In these latter conditions, participants using task-interleaving strategies of response organization were not only able to minimize dual-task costs but even to achieve a benefit of multitasking in terms of higher task throughput than in single-task conditions. For switchers, this was already the case when the processing codes are separated at the perceptual-cognitive processing level (Experiment 2), and even increased with further resource separation. For response grouper it applied to the two conditions with separated response modalities. These findings challenge the consensus often derived from research with task-switching or the PRP paradigm that concurrent performance of discrete cognitive tasks generally leads to cost effects and, thus, 
that multitasking cannot be efficient and should be avoided (see Kiesel et al., 2010; Koch et al., 2018; Pashler, 2000, for reviews). The main reason for these multitasking benefits could be identified in a higher degree and efficiency of overlapping processing of the tasks in the dual-task conditions with less resource competition. This sort of task processing led to timegains particularly at task switches or in periods before the execution of grouped responses. Even though, these time gains could not be realized with all task-switches or response groupings, they obviously more than compensated any remaining costs related to task-set reconfiguration (Monsell, 2003) or new costs arising from self-organized multitasking (Brüning et al., 2020) when accumulated across time.

As mentioned in the discussion of the results of Experiment 2, the finding that taskinterleaving strategies combined with overlapping processing of tasks are particularly effective if the tasks to be performed concurrently do not compete for the same processingcodes needed for perceptual-cognitive and/or response-related processes supports basic assumptions of the MRT (Wickens, 1980, 1984, 2002). However, the results of the present study also suggest that this general prediction of MRT needs to be differentiated, as the effects caused by a separation of the processing codes differed greatly for the two processing stages. Just a separation of processing codes at the stage of perceptual-cognitive processing only led to relatively weak improvements. These were statistically significant in the second experiment only and remained restricted to the switching strategy. In contrast, the separation of processing-code resources with respect to response modalities (manual vs. vocal) had a much stronger influence on the multitasking efficiency of both interleaving strategies. Actually, the gain of multitasking efficiency achieved by separating the response modalities alone was almost as large as in the dual-task condition with minimal resource competition. Moreover, especially for response grouper, a substantial gain in multitasking performance instead of considerable costs was not observed before the response modalities of the two tasks got separated. This suggests, that a separation of resources in terms of processing codes 
needed for response-related processes might be much more effective than a separation of processing-code resources needed for the perceptual-cognitive stage. The specific mechanism involved is somewhat difficult to be revealed based on the current data, though. One possibility is to assume that it was not just the effect of a separation of response modalities per se but the result of a specific interaction effect between the different types of processing code resources, which contributed to the improvement of multitasking efficiency in case of response-modality separation. This is suggested by the theory of event coding (Hommel et al., 2001; Prinz, 1990; see also and specifically the findings of Göthe et al., 2016 and Schaeffner et al., 2018). According to TEC, tasks involving spatial-manual and verbal-vocal stimulusresponse mappings should be especially compatible with respect to internal processing pathways resulting in low dual-task interference. Our condition of minimal resource competition in the second experiment was directly optimized for this purpose. However, the condition with just a separation of response modalities included also at least one such task (verbal - vocal), whereas the other task was incompatible (verbal/manual). The boost of multitasking efficiency in the latter condition then would suggest that dual-task costs can already be reduced if just one of the tasks to be performed concurrently has a compatible resource pairing in terms of TEC. However, it seems implausible to assume that the efficiency gains achieved by just one task being compatible in this respect can be as high as for a condition with two compatible tasks, as in our second experiment. Furthermore, it would be difficult to explain, why particularly the response-grouping strategy benefitted from such compatibility effect. Thus, we assume that the particular effect of separating the response modalities in our second experiment might rather be attributed to a reduction of structural interferences between the two hands achieved by decoupling the response modes. Such structural interference effects are known from research with bimanual responses (Heuer, 1990; Spijkers \& Heuer, 1995, 2004) and might also have masked clearer effects of the 
separation of processing codes just at the perceptual-cognitive stage and particularly produced conflicts for the response grouping strategy in these dual-tasks.

\section{Conclusions, Limitations and Prospects}

To conclude, the results of the present study challenge the simplistic view often found in public media (Bradberry, 2014; MacMillan, 2016) that multitasking is inherently inefficient and provide a more differentiated picture of the circumstances under which multitasking can or cannot be optimal. In this sense, the current data illustrate the importance of an individual differences approach to determine whether the characteristics of the respective tasks have a detrimental or beneficial effect on multitasking performance. Specifically, an individual preference for interleaving strategies benefited from less task similarity in terms of multiple available resources. Furthermore, the presented data suggests that the strategies of response organization are comparatively stable and not as flexibly adapted to varying task characteristics as the preference for modes of task processing.

Of course, the current study also has some limitations. First, we just investigated effects of a separation of resource demands on the dimension of processing codes (Wickens, 2002). Future studies might even include other dimensions. For example, Schaeffner et al. (2018) showed that in addition to the compatibility of stimulus-response features also a consideration of the compatibility of stimulus modality and response modality can contribute to a reduction of task-switching costs. Accordingly, a further separation of the stimulus modality with compatible stimulus-response mappings (e.g., auditory-verbal-vocal vs. visualspatial-manual) might even further improve the multitasking efficiency of interleaving strategies. Due to the specific characteristics of auditory stimuli, such advanced effects could not be addressed based on the FCDT paradigm. Given the temporary nature of auditory presentation of, e.g., verbal stimuli, they inevitably contain an inviting character, which would have biased the strategy of response organization towards immediate responding to these stimuli. Alternatively, we had to work with a constant repetition of auditory stimuli, which 
probably would have raised similar issues. Thus, other paradigms are needed to further investigate how task organization in self-organized multitasking is affected by specific compatibility relations of input-modality and processing-code resources. Second, given that we always provided a visual preview to the stimulus of the task currently not performed, which represents a basic characteristic of the FCDT paradigm, our results are only valid for multitasking situations where this is possible. However, there are certainly multitasking situations, which do not provide such preview. It would be interesting to see whether preferences for blocking, switching and response grouping also emerge in such situations, given that the advantages of interleaving strategies cannot be used. Based on the results of the conditions with maximal resource competition in the two experiments we would assume that the same preferences could be found but this remains to be seen. Finally, the present results indicate that the improved efficiency of interleaving strategies under conditions of lowered resource competition results from overlapping processing of the two tasks but do not provide any insights in what actually was processed in an overlapping manner. In this vein, it would be of some interest to further determine when and how exactly overlapping takes place, that is, which stages of information processing are involved. Thus, also this issue remains to be resolved by future studies.

\section{Compliance with Ethical Standards}

Conflict of Interest: Dietrich Manzey, Jovita Brüning, and Marie Mückstein declare that they have no conflict of interest.

Ethical approval: All procedures performed in studies involving human participants were in accordance with the ethical standards of the institutional and/or national research committee and with the 1964 Helsinki declaration and its later amendments or comparable ethical standards. This article does not contain any studies with animals performed by the authors. Informed consent: Informed consent was obtained from all individual participants included in the study. 


\section{Appendix: Definition of Overall Dual-Tasking Performance Efficiency (ODTPE)}

The ODTPE measure describes how often two tasks can be answered correctly in a given time when a person has to complete these tasks simultaneously or in close succession, compared with performance on the respective tasks under single-task conditions. Considering only the throughput of correct responses, it generates an overall measure of dual-tasking efficiency that represents multitasking costs or benefits reflected in both, speed and accuracy.

In the experiments presented in the present paper, single-task blocks lasted half as long (i.e., one minute) as dualtask blocks (two minutes $)^{3}$. For this case the measure is formally defined as follows:

$$
O D T P E=100 *\left[\frac{n C_{\mathrm{A} \_ \text {dual }}}{n C_{\mathrm{A} \_ \text {single }}}+\frac{n C_{\mathrm{B} \_ \text {dual }}}{n C_{\mathrm{B} \_ \text {single }}}\right]-100,
$$

with $n C_{A_{-} \text {single }}$ and $n C_{B_{-} \text {single }}$ defined as number of correct responses in the respective tasks under single-task conditions, and $n C_{A_{-} d u a l}$ and $n C_{B_{-} \text {dual }}$ defined as the corresponding performance in dual-task conditions. ODTPE values of 0 reflect neither dual-tasking benefits nor costs. That is, the throughput of correctly performed tasks does not change in dual-task compared to single-task conditions. In contrast, overall dual-tasking benefits and costs are indicated by positive and negative ODTPE scores, respectively.

The basic logic of this measure can be illustrated by the following Gedankenexperiment: Assume an individual can correctly solve 60 trials of a given task A, and 80 trials of a given task B, both under single-task conditions in one-minute blocks. Imagine now that this individual then has to work on both tasks concurrently for two minutes in a dual-task condition. Given that the individual is supposed to set equal priority on both tasks, again approximately one minute is available for each task. In case the participant is not able to gain any benefits from dual-tasking (e.g., from overlapping processing) or has any time costs (e.g., the coordination of the two tasks), one would expect that the throughput of correct tasks is essentially the same in dual-task compared to single-task conditions, i.e., 60 trials of task A and 80 trials of task B in the two-minute dual-task block, resulting in an ODTPE score of 0 . However, if any dual-task costs arise in terms of extra time needed to perform the two tasks concurrently or in close succession, this should be reflected in a lesser task throughput in dual-task than single-task conditions, reflected in an ODTPE score $<0$. Finally, in case the individual would be able to save time when performing both tasks in a dual-tasking setting, e.g., by being able to integrate or parallelize the processing of both tasks to some extent, this would result in a higher task throughput in dual-task compared to single-task conditions yielding an ODTPE score $>0$.

\footnotetext{
${ }^{3}$ Note that in the calculations included in this study, the two dual-task blocks included in one run are compared separately with the corresponding subsequent single-task blocks, maintaining the same relations described here.
} 
References

Allport, A., Styles, E. A., \& Hsieh, S. (1994). Shifting intentional set: Exploring the dynamic control of tasks. In C. Umiltà \& M. Moscovitch (Eds.), Attention and Performance XV: Conscious and nonconscious informing processing (pp. 421-452). A Bradford Book; The MIT Press.

Arrington, C. M., \& Logan, G. D. (2004). The cost of a voluntary task switch. Psychological Science, 15(9), 610-615. https://doi.org/10.1111/j.0956-7976.2004.00728.x

Arrington, C. M., \& Logan, G. D. (2005). Voluntary task switching: Chasing the elusive homunculus. Journal of Experimental Psychology: Learning, Memory, and Cognition, 31(4), 683-702. https://doi.org/10.1037/0278-7393.31.4.683

Baddeley, A. D. (1986). Working Memory (11th ed.). Oxford University Press.

Baddeley, A. D. (2002). Is Working Memory Still Working? European Psychologist, 7(2), 85-97. https://doi.org/10.1027//1016-9040.7.2.85

Baddeley, A. D. (2012). Working memory: Theories, models, and controversies. Annual Review of Psychology, 63, 1-29. https://doi.org/10.1146/annurev-psych-120710-100422

Bradberry, T. (2014, October 8). Multitasking Damages Your Brain And Career, New Studies Suggest. https://www.forbes.com/sites/travisbradberry/2014/10/08/multitasking-damagesyour-brain-and-career-new-studies-suggest/\#16addf1956ee

Braem, S., Bugg, J. M., Schmidt, J. R., Crump, M. J., Weissman, D. H., Notebaert, W., \& Egner, T. (2019). Measuring adaptive control in conflict tasks. Trends in Cognitive Sciences, 23(9), 769-783.

Brüning, J., \& Manzey, D. (2018). Flexibility of individual multitasking strategies in taskswitching with preview: Are preferences for serial versus overlapping task processing dependent on between-task conflict? Psychological Research, 82(1), 92-108. https://doi.org/10.1007/s00426-017-0924-0 
Brüning, J., Reissland, J., \& Manzey, D. (2020). Individual preferences for task coordination strategies in multitasking: Exploring the link between preferred modes of processing and strategies of response organization. Psychological Research. Advance online publication. https://doi.org/10.1007/s00426-020-01291-7

Caird, J. K., Willness, C. R., Steel, P., \& Scialfa, C. (2008). A meta-analysis of the effects of cell phones on driver performance. Accident; Analysis and Prevention, 40(4), 1282-1293. https://doi.org/10.1016/j.aap.2008.01.009

Conway, A. R. A., Kane, M. J., Bunting, M. F., Hambrick, D. Z [D. Zach], Wilhelm, O., \& Engle, R. W. (2005). Working memory span tasks: A methodological review and user's guide. Psychonomic Bulletin \& Review, 12(5), 769-786.

https://doi.org/10.3758/BF03196772

Damos, D. L., Smist, T. E., \& Bittner, A. C. (1983). Individual differences in multiple-task performance as a function of response strategy. Human Factors: The Journal of the Human Factors and Ergonomics Society, 25(2), pp. 215-226.

http://hfs.sagepub.com/content/25/2/215.full.pdf+html

Damos, D. L., \& Wickens, C. D. (1980). The identification and transfer of timesharing skills. Acta Psychologica, 46(1), 15-39. https://doi.org/10.1016/0001-6918(80)90057-8

De Jong, R. (1995). The role of preparation in overlapping-task performance. The Quarterly Journal of Experimental Psychology Section a, 48(1), 2-25. https://doi.org/10.1080/14640749508401372

Engle, R. W. (2002). Working memory capacity as executive attention. Current Directions in Psychological Science, 11(1), 19-23.

Engle, R. W., \& Kane, M. J. (2004). Exectutive attention, working memory capactiy, and a two-factor theory of cognitive control. In B. H. Ross (Ed.), The psychology of learning and motivation (pp. 145-200). Elsevier Academic. 
Faul, F., Erdfelder, E., Buchner, A., \& Lang, A.-G. (2009). Statistical power analyses using G*Power 3.1: Tests for correlation and regression analyses. Behavior Research Methods, 41(4), 1149-1160. https://doi.org/10.3758/BRM.41.4.1149

Fischer, R., \& Plessow, F. (2015). Efficient multitasking: Parallel versus serial processing of multiple tasks. Frontiers in Psychology, 6(Suppl. 1), 112. https://doi.org/10.3389/fpsyg.2015.01366

Fried, P. J., Rushmore, R. J., Moss, M. B., Valero-Cabré, A., \& Pascual-Leone, A. (2014). Causal evidence supporting functional dissociation of verbal and spatial working memory in the human dorsolateral prefrontal cortex. The European Journal of Neuroscience, 39(11), 1973-1981. https://doi.org/10.1111/ejn.12584

Friedman, N. P., \& Miyake, A. (2005). Comparison of four scoring methods for the reading span test. Behavior Research Methods, 37(4), 581-590. https://doi.org/10.3758/BF03192728

Fröber, K., \& Dreisbach, G. (2017). Keep flexible - Keep switching! The influence of forced task switching on voluntary task switching. Cognition, 162, 48-53. https://doi.org/10.1016/j.cognition.2017.01.024

Ganis, G., \& Kievit, R. (2015). A New Set of Three-Dimensional Shapes for Investigating Mental Rotation Processes: Validation Data and Stimulus Set, 3(1). https://doi.org/10.5334/jopd.ai

Göthe, K., Oberauer, K., \& Kliegl, R. (2016). Eliminating dual-task costs by minimizing crosstalk between tasks: The role of modality and feature pairings. Cognition, 150, 92-108. https://doi.org/10.1016/j.cognition.2016.02.003

Hartigan, J. A., \& Hartigan, P. M. (1985). The dip test of unimodality. The Annals of Statistics, 13(1), 70-84. https://doi.org/10.1214/aos/1176346577 
Herdman, C. M., \& Friedman, A. (1985). Multiple Resources in Divided Attention: A CrossModal Test of the Independence of Hemispheric Resources. Journal of Experimental Psychology. Human Perception and Performance, 11(1), 40-49.

Heuer, H. (1990). Rapid responses with the left or right hand: response-response compatibility effects due to intermanual interactions. In R. W. Proctor \& T. G. Reeve (Eds.), Advances in psychology: Vol. 65. Stimulus-response compatibility: An integrated perspective (Vol. 65, pp. 311-342). North-Holland; Distributors for the U.S. and Canada, Elsevier Science Pub. Co.

Hommel, B. (2015). Between persistence and flexibility: The Yin and Yang of action control. In A. J. Elliot (Ed.), Advances in Motivation Science (Vol. 2, pp. 33-67). Elsevier. https://doi.org/10.1016/bs.adms.2015.04.003

Hommel, B., Müsseler, J., Aschersleben, G., \& Prinz, W [W.] (2001). The Theory of Event Coding (TEC): A framework for perception and action planning. Behavioral and Brain Sciences, 24(5), 849-78; discussion 878-937. https://doi.org/10.1017/s0140525x01000103

Horrey, W. J., \& Wickens, C. D. (2006). Examining the Impact of Cell Phone Conversations on Driving Using Meta-Analytic Techniques. Human Factors, 48(1), 196-205.

Howell, D. C. (2016). Fundamental statistics for the behavioral sciences (9th ed.). Cengage Learning.

Janssen, C. P., Brumby, D. P., Dowell, J., Chater, N., \& Howes, A. (2011). Identifying Optimum Performance Trade-Offs Using a Cognitively Bounded Rational Analysis Model of Discretionary Task Interleaving. Topics in Cognitive Science, 3(1), 123-139. https://doi.org/10.1111/j.1756-8765.2010.01125.x

Jersild, A. T. (1927). Mental set and shift. Archives of Psychology, 89.

Kane, M. J., Conway, A. R. A., Hambrick, D. Z [David Z.], \& Engle, R. W. (2007). Variation in Working Memory Capacity as Variation in Executive Attention and Control. In A. R. A. 
Conway, C. Jarrold, M. J. Kane, A. Miyake, \& J. N. Towse (Eds.), Variation in Working Memory (pp. 21-48). Oxford University Press.

Kessler, Y., Shencar, Y., \& Meiran, N. (2009). Choosing to switch: Spontaneous task switching despite associated behavioral costs. Acta Psychologica, 131(2), 120-128. https://doi.org/10.1016/j.actpsy.2009.03.005

Kiesel, A., Steinhauser, M., Wendt, M., Falkenstein, M., Jost, K., Philipp, A. M., \& Koch, I. (2010). Control and interference in task switching-A review. Psychological Bulletin, 136(5), 849-874. https://doi.org/10.1037/a0019842

Koch, I., \& Allport, A. (2006). Cue-based preparation and stimulus-based priming of tasks in task switching. Memory \& Cognition, 34(2), 433-444. https://doi.org/10.3758/BF03193420

Koch, I., Poljac, E., Müller, H., \& Kiesel, A. (2018). Cognitive structure, flexibility, and plasticity in human multitasking - An integrative review of dual-task and task-switching research. Psychological Bulletin, 144(6), 557-583. https://doi.org/10.1037/bul0000144

Kübler, S., Reimer, C. B., Strobach, T., \& Schubert, T. (2018). The impact of free-order and sequential-order instructions on task-order regulation in dual tasks. Psychological Research, 82(1), 40-53. https://doi.org/10.1007/s00426-017-0910-6

Kushleyeva, Y., Salvucci, D. D., \& Lee, F. J. (2005). Deciding when to switch tasks in timecritical multitasking. Cognitive Systems Research, 6(1), 41-49. https://doi.org/10.1016/j.cogsys.2004.09.005

Lehle, C., \& Hübner, R. (2009). Strategic capacity sharing between two tasks: evidence from tasks with the same and with different task sets. Psychological Research Psychologische Forschung, 73(5), 707-726. https://doi.org/10.1007/s00426-008-0162-6

MacMillan, A. (2016, July 4). 12 Reasons to Stop Multitasking Now! https://www.health.com/condition/adhd/12-reasons-to-stop-multitasking-now 
Mair, P., \& Wilcox, R. R. (2019). Robust statistical methods in R using the WRS2 package. Behavior Research Methods, Forthcoming.

Maquestiaux, F., Lague-Beauvais, M., Ruthruff, E., \& Bherer, L. (2008). Bypassing the central bottleneck after single-task practice in the psychological refractory period paradigm: Evidence for task automatization and greedy resource recruitment. Memory \& Cognition, 36(7), 1262-1282. https://doi.org/10.3758/MC.36.7.1262

Maquestiaux, F., Ruthruff, E., Defer, A., \& Ibrahime, S. (2018). Dual-task automatization: The key role of sensory-motor modality compatibility. Attention, Perception \& Psychophysics, 80(3), 752-772. https://doi.org/10.3758/s13414-017-1469-4

Mccart, A. T., Hellinga, L. A., \& Bratiman, K. A. (2006). Cell phones and driving: review of research. Traffic Injury Prevention, 7(2), 89-106.

McLeod, P. (1977). A dual task response modality effect: Support for multiprocessor models of attention. Quarterly Journal of Experimental Psychology, 29(4), 651-667.

Mittelstädt, V., Dignath, D., Schmidt-Ott, M., \& Kiesel, A. (2018). Exploring the repetition bias in voluntary task switching. Psychological Research, 82(1), 78-91. https://doi.org/10.1007/s00426-017-0911-5

Mittelstädt, V., Miller, J., \& Kiesel, A. (2019). Linking task selection to task performance: Internal and predictable external processing constraints jointly influence voluntary task switching behavior. Journal of Experimental Psychology. Human Perception and Performance, 45(12), 1529-1548. https://doi.org/10.1037/xhp0000690

Monsell, S. (1996). Control of mental processes. In V. Bruce (Ed.), Unsolved mysteries of the mind (pp. 93-148). Erlbaum.

Monsell, S. (2003). Task switching. Trends in Cognitive Sciences, 7(3), 134-140. https://doi.org/10.1016/S1364-6613(03)00028-7 
Mueller, S. T. (2012). The Psychology Experiment Building Language, Version 0.13. http://pebl.sourceforge.net

Mueller, S. T., \& Piper, B. J. (2014). The Psychology Experiment Building Language (PEBL) and PEBL Test Battery. Journal of Neuroscience Methods, 222, 250-259. https://doi.org/10.1016/j.jneumeth.2013.10.024

Navon, D., \& Gopher, D. (1979). On the economy of the human-processing system. Psychological Review, 86(3), 214-255. https://doi.org/10.1037/0033-295X.86.3.214

Oberauer, K., Lewandowsky, S., Awh, E., Brown, G. D. A., Conway, A., Cowan, N., Donkin, C., Farrell, S., Hitch, G. J., Hurlstone, M. J., Ma, W. J., Morey, C. C., Nee, D. E., Schweppe, J., Vergauwe, E., \& Ward, G. (2018). Benchmarks for models of short-term and working memory. Psychological Bulletin, 144(9), 885-958. https://doi.org/10.1037/bul0000153

Parry, D. A., \& Le Roux, D. B. (2019). Media multitasking and cognitive control: A systematic review of interventions. Computers in Human Behavior(92), 316-327.

Pashler, H. (1994a). Dual-task interference in simple tasks: Data and theory. Psychological Bulletin, 116(2), 220-244. https://doi.org/10.1037/0033-2909.116.2.220

Pashler, H. (1994b). Graded capacity-sharing in dual-task interference? Journal of Experimental Psychology: Human Perception and Performance, 20, 330-342.

Pashler, H. (2000). Task switching and multitask performance. In S. Monsell \& J. Driver (Eds.), Control of cognitive processes: Attention and Performance XVIII (pp. 277-307). MIT Press.

Pashler, H., \& Johnston, J. C. (1989). Chronometric evidence for central postponement in temporally overlapping tasks. The Quarterly Journal of Experimental Psychology Section a, 41(1), 19-45. https://doi.org/10.1080/14640748908402351 
Patera, W. (2018). Different $5 x 5$ pixel-patterns used for surface tracking in eye-tracking. https://github.com/pupil-labs/pupilhelpers/blob/master/markers_stickersheet/all_markers.png

Payne, S. J., Duggan, G. B., \& Neth, H. (2007). Discretionary task interleaving: Heuristics for time allocation in cognitive foraging. Journal of Experimental Psychology: General, 136(3), 370-388. https://doi.org/10.1037/0096-3445.136.3.370

Polson, M. C., \& Friedman, A. (1988). Task-Sharing within and between Hemispheres: A Multiple-Resources Approach. Human Factors, 30(5), 633-643. https://doi.org/10.1177/001872088803000507

Poposki, E. M., \& Oswald, F. L. (2010). The Multitasking Preference Inventory: Toward an Improved Measure of Individual Differences in Polychronicity. HUMAN PERFORMANCE, 23(3), 247-264. https://doi.org/10.1080/08959285.2010.487843

Prinz, W [W.]. (1990). A Common Coding Approach to Perception and Action. In O. Neumann \& W. Prinz (Eds.), Relationships Between Perception and Action: Current Approaches (pp. 167-201). Springer Berlin Heidelberg. https://doi.org/10.1007/978-3-64275348-0_7

Redick, T. S., Broadway, J. M., Meier, M. E., Kuriakose, P. S., Unsworth, N., Kane, M. J., \& Engle, R. W. (2012). Measuring Working Memory Capacity With Automated Complex Span Tasks. European Journal of Psychological Assessment, 28(3), 164-171. https://doi.org/10.1027/1015-5759/a000123

Reissland, J., \& Manzey, D. (2016). Serial or overlapping processing in multitasking as individual preference: Effects of stimulus preview on task switching and concurrent dualtask performance. Acta Psychologica, 168, 27-40. https://doi.org/10.1016/j.actpsy.2016.04.010 
Rogers, R. D., \& Monsell, S. (1995). Costs of a predictable switch between simple cognitive tasks. Journal of Experimental Psychology: General, 124(2), 207-231. https://doi.org/10.1037/0096-3445.124.2.207

Ruthruff, E., van Selst, M., Johnston, J. C., \& Remington, R. (2006). How does practice reduce dual-task interference: Integration, automatization, or just stage-shortening? Psychological Research Psychologische Forschung, 70(2), 125-142. https://doi.org/10.1007/s00426-004-0192-7

Salvucci, D. D. (2013). Chapter 3: Multitasking. In J. D. Lee \& A. Kirlik (Eds.), The Oxford handbook of cognitive engineering. Oxford University Press.

Schaeffner, S., Koch, I., \& Philipp, A. M. (2018). Sensory-motor modality compatibility in multitasking: The influence of processing codes. Acta Psychologica, 191, 210-218. https://doi.org/10.1016/j.actpsy.2018.09.012

Schumacher, E. H., Seymour, T. L., Glass, J. M., Fencsik, D. E., Lauber, E. J., Kieras, D. E., \& Meyer, D. E. (2001). Virtually perfect time sharing in dual-task performance: Uncorking the central cognitive bottleneck. Psychological Science, 12(2), 101-108.

Slocombe, T. E., \& Bluedorn, A. C. (1999). Organizational behavior implications of the congruence between preferred polychronicity and experienced work-unit polychronicity. Journal of Organizational Behavior, 20, 75-99.

Smith, E. E., Jonides, J., \& Koeppe, R. A. (1996). Dissociating verbal and spatial working memory using pet. Cerebral Cortex, 6(1), 11-20.

Spijkers, W., \& Heuer, H. (1995). Structural Constraints on the Performance of Symmetrical Bimanual Movements with Different Amplitudes. The Quarterly Journal of Experimental Psychology Section a, 48(3), 716-740. https://doi.org/10.1080/14640749508401412

Spijkers, W., \& Heuer, H. (2004). Behavioral principles of interlimb coordination. In S. P. Swinnen \& J. Duysens (Eds.), Neuro-Behavioral Determinants of Interlimb Coordination: 
A multidisciplinary approach (pp. 223-258). Springer Science + Business Media, LLC. 10.1007/978-1-4419-9056-3_9

Strobach, T., Schubert, T., Pashler, H., \& Rickard, T. (2014). The specificity of learned parallelism in dual-memory retrieval. Memory \& Cognition, 42(4), 552-569. https://doi.org/10.3758/s13421-013-0382-X

Telford, C. W. (1931). The refractory phase of voluntary and associative responses. Journal of Experimental Psychology, 14(1), 1-36. https://doi.org/10.1037/h0073262

Tombu, M., \& Jolicœur, P. (2003). A central capacity sharing model of dual-task performance. Journal of Experimental Psychology: Human Perception and Performance, 29(1), 3-18. https://doi.org/10.1037/0096-1523.29.1.3

Tsang, P. S. (2006). Regarding time-sharing with convergent operations. Acta Psychologica, 121(2), 137-175. https://doi.org/10.1016/j.actpsy.2005.07.002

Turner, M. L., \& Engle, R. W. (1989). Is working memory capacity task dependent? Journal of Memory and Language, 28(2), 127-154. https://doi.org/10.1016/0749-596X(89)90040-5

Ulrich, R., \& Miller, J. (2008). Response grouping in the psychological refractory period (PRP) paradigm: Models and contamination effects. Cognitive Psychology, 57(2), 75-121. https://doi.org/10.1016/j.cogpsych.2007.06.004

Unsworth, N., Heitz, R., Schrock, J. C., \& Engle, R. W. (2005). An automated version of the operation span task. Behavior Research Methods, 37(3), 498-505. https://doi.org/10.3758/BF03192720

van der Schuur, W. A., Baumgartner, S. E., Sumter, S. R., \& Valkenburg, P. M. (2015). The consequences of media multitasking for youth: A review. Computers in Human Behavior, 53, 204-215. https://doi.org/10.1016/j.chb.2015.06.035

Welford, A. T. (1952). The "psychological refractory period" and the timing of high-speed performance - A review and a theory. British Journal of Psychology, 43, 2-19. 
Wickens, C. D. (1980). The structure of attentional resources. In R. Nickerson (Ed.), Attention and performance VIII (pp. 239-258). Lawrence Erlbaum Associates Inc.

Wickens, C. D. (1984). Engineering psychology and human performance. Merrill.

Wickens, C. D. (2002). Multiple resources and performance prediction. Theoretical Issues in Ergonomics Science, 3(2), 159-177. https://doi.org/10.1080/14639220210123806

Wickens, C. D. (2008). Multiple resources and mental workload. Human Factors, 50(3), 449455. https://doi.org/10.1518/001872008X288394

Wickens, C. D., Hollands, J. G., Banbury, S., \& Parasuraman, R. (2013). Multitasking Corrected. In C. D. Wickens, J. G. Hollands, S. Banbury, \& R. Parasuraman (Eds.), Engineering psychology and human performance (pp. 321-345). Pearson.

Wickens, C. D., \& Liu, Y. (1988). Codes and Modalities in Multiple Resources: A Success and a Qualification. Human Factors, 30(5), 599-616.

Wickens, C. D., \& Sandry, D. L. (1982). Task-hemispheric integrity in dual task performance. Acta Psychologica, 52, 227-247.

Wickens, C. D., Sandry, D. L., \& Vidulich, M. (1983). Compatibility and Resource Competition between Modalities of Input, Central Processing, and Output. Human Factors, 25(2), 227-248. 